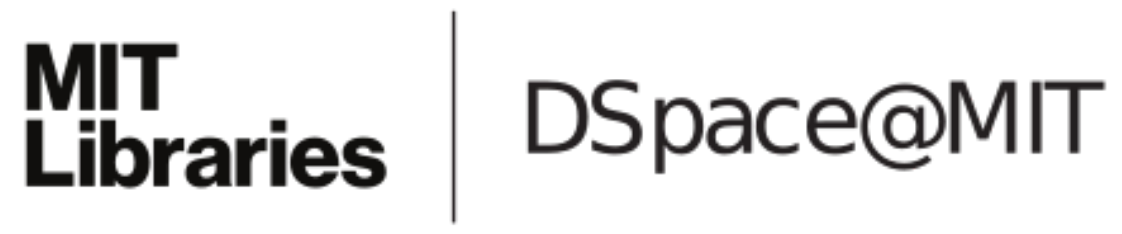

MIT Open Access Articles

Nanoemulsions: formation, properties and applications

The MIT Faculty has made this article openly available. Please share how this access benefits you. Your story matters.

Citation: Gupta, Ankur et al. “Nanoemulsions: Formation, Properties and Applications.” Soft Matter 12.11 (2016): 2826-2841. (C) 2016 Royal Society of Chemistry

As Published: http://dx.doi.org/10.1039/c5sm02958a

Publisher: Royal Society of Chemistry

Persistent URL: http://hdl.handle.net/1721.1/107439

Version: Final published version: final published article, as it appeared in a journal, conference proceedings, or other formally published context

Terms of use: Creative Commons Attribution-NonCommercial 3.0 Unported 


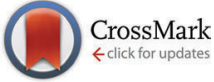

Cite this: Soft Matter, 2016, 12,2826

Received 7th December 2015, Accepted 19th February 2016

DOI: $10.1039 / \mathrm{c} 5 \mathrm{sm} 02958 \mathrm{a}$

www.rsc.org/softmatter

\title{
Nanoemulsions: formation, properties and applications
}

\begin{abstract}
Ankur Gupta, ${ }^{a}$ H. Burak Eral, ${ }^{\text {bc }}$ T. Alan Hatton ${ }^{a}$ and Patrick S. Doyle*a
Nanoemulsions are kinetically stable liquid-in-liquid dispersions with droplet sizes on the order of $100 \mathrm{~nm}$. Their small size leads to useful properties such as high surface area per unit volume, robust stability, optically transparent appearance, and tunable rheology. Nanoemulsions are finding application in diverse areas such as drug delivery, food, cosmetics, pharmaceuticals, and material synthesis. Additionally, they serve as model systems to understand nanoscale colloidal dispersions. High and low energy methods are used to prepare nanoemulsions, including high pressure homogenization, ultrasonication, phase inversion temperature and emulsion inversion point, as well as recently developed approaches such as bubble bursting method. In this review article, we summarize the major methods to prepare nanoemulsions, theories to predict droplet size, physical conditions and chemical additives which affect droplet stability, and recent applications.
\end{abstract}

\section{Introduction}

Nanoemulsions are emulsions with droplet size on the order of $100 \mathrm{~nm}$. A typical nanoemulsion contains oil, water and an emulsifier. The addition of an emulsifier is critical for the creation of small sized droplets as it decreases the interfacial tension i.e., the surface energy per unit area, between the oil

${ }^{a}$ Massachusetts Institute of Technology, Cambridge, MA, USA.

E-mail: pdoyle@mit.edu; Fax: +1 617324 0066; Tel: +1 6172534534

${ }^{b}$ Delft University of Technology, The Netherlands

${ }^{c}$ Utrecht University, The Netherlands

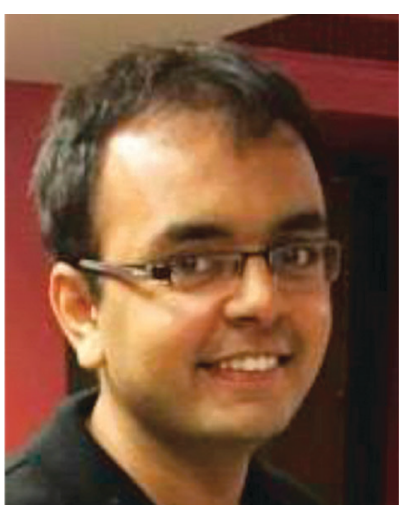

Ankur Gupta
Ankur Gupta is a PhD candidate at Massachusetts Institute of Technology in the Chemical Engineering department working with Prof. Patrick S. Doyle and Prof. T. Alan Hatton. He is currently working on nanoemulsions and their applications. His research interests are interfacial science, multiphase fluid flow and microfluidics. and water phases of the emulsion. The emulsifier also plays a role in stabilizing nanoemulsions through repulsive electrostatic interactions and steric hindrance. ${ }^{1}$ The emulsifier used is generally a surfactant, but proteins and lipids have also been effective in the preparation of nanoemulsions. ${ }^{2-12}$ Over the past decade or more, the research focus has been on preparing nanoemulsions through various methods, broadly classified into two primary categories: high-energy and low-energy methods. ${ }^{13-15}$ High energy methods such as high pressure homogenization $(\mathrm{HPH})$ and ultrasonication ${ }^{15}$ consume significant energy $\left(\sim 10^{8}-10^{10} \mathrm{~W} \mathrm{~kg}^{-1}\right)$ to make small droplets. On the other hand, low energy methods exploit specific system properties
H. Burak Eral

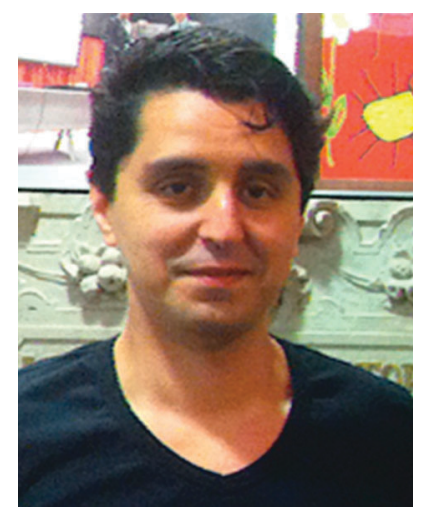

H. Burak Eral Fluids group in University of Twente in 2012. His research spans interdisciplinary fields including: soft matter, process intensification, crystallization, hydrodynamics, rheology and wetting physics. 
to make small droplets without consuming significant energy $\left(\sim 10^{3} \mathrm{~W} \mathrm{~kg}^{-1}\right)$. Phase inversion temperature $(\mathrm{PIT})^{16}$ and emulsion inversion point (EIP) $)^{17-19}$ are two examples of low energy approaches for the formation of nanoemulsions. Recently, a few novel technologies such as bubble bursting at oil/water interface ${ }^{20}$ and evaporative ripening ${ }^{21}$ have also been developed for making nanoemulsions. The second section of this article reviews the various methods to make nanoemulsions. We also discuss the ways to control and predict droplet size based on system properties and process parameters.

Nanoemulsions are kinetically stable, i.e., given sufficient time, a nanoemulsion phase separates..$^{22,23}$ In Section 3, we discuss the underlying physics behind destabilization mechanisms such as flocculation, coalescence, Ostwald ripening and creaming. We show that Ostwald ripening is the dominant destabilization mechanism for nanoemulsions. We also review the literature on the effect of parameters like nanoemulsion composition and temperature on destabilization rates of nanoemulsions. ${ }^{2,16,18,24-29}$ A short discussion on the trapped species method for making stable nanoemulsions is also presented.

The range of nanoemulsion applications spans diverse fields including drug delivery, ${ }^{30-62}$ where $\mathrm{O} / \mathrm{W}$ nanoemulsions have been used to deliver hydrophobic drugs; the food industry, where flavored nanoemulsions with improved curcumin/ $\beta$-carotene and digestibility have been prepared $;^{5-7,9-12,63-73}$ and in cosmetic industry where nanoemulsions have been tested for skin hydration and ease of application. ${ }^{15,74,75}$ Researchers have also showed that many problems faced in current methods of pharmaceutical crystallization processes can be avoided with nanoemulsions. ${ }^{76,77}$ Nanoemulsions have also been used as building blocks for complex material synthesis such as compartmentalized nanoparticles and encapsidated oil droplets. ${ }^{15,78-81}$ The fourth section of this paper discusses important properties on nanoemulsions and discusses a wide range of nanoemulsion applications.

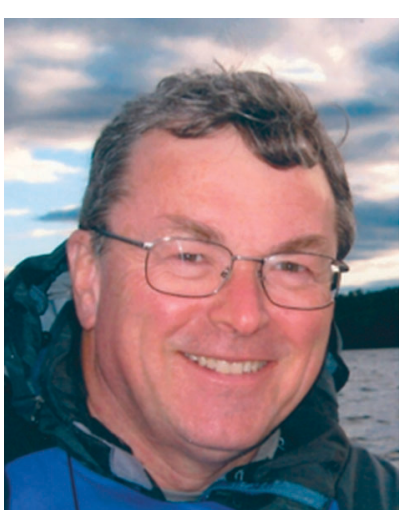

T. Alan Hatton
T. Alan Hatton is the Ralph Landau Professor of Chemical Engineering and Director of the David H. Koch School of Chemical Engineering Practice at the Massachusetts Institute of Technology. He received his $P h D$ in Chemical Engineering in 1981 from University of Wisconsin. His group's research focuses on the synthesis, understanding and application of stimuli-responsive materials, which include nanoparticles, nanofibers, surfactants, polymers and gels, with a current focus on the development of electrochemical processes to facilitate chemical separations and to mediate the transformation of captured waste to useful commodity chemicals.
There is some confusion in the literature regarding a precise definition of nanoemulsions which are often confused with the thermodynamically stable microemulsions which form spontaneously. ${ }^{22,23}$ The major differences between classical emulsions (or macroemulsions), nanoemulsions and microemulsions is in droplet size range and stability characteristics, as summarized in Fig. 1. Macroemulsions and nanoemulsions are both thermodynamically unstable, i.e. given sufficient time, phase separation occurs. However, because of the small size of nanoemulsions (sometimes also referred as 'miniemulsions'), nanoemulsions can be kinetically stable over long time scales. Nanoemulsion metastability has nothing to do with proximity to an equilibrium state. ${ }^{2}$ On the other hand, since microemulsions are thermodynamically stable systems in equilibrium, they are sensitive to changes in temperature and composition. Therefore, nanoemulsions are attractive for aforementioned applications because they are relatively the least sensitive to physical and chemical changes. Two recent studies have clarified the distinction between nanoemulsions and microemulsions, and the interested readers are referred to these reports that detail the differences between these two classes of liquid-inliquid dispersions. ${ }^{22,23}$

\section{Formation}

An understanding of the physics of nanoemulsion formation is critical for the control of nanoemulsion droplet size. Nanoemulsions are typically prepared in a two-step process where a macroemulsion is first prepared, and is then converted to a nanoemulsion in a second step. In this section, we describe various methods developed over the past decade or so to prepare nanoemulsions, and indicate the progress made on the prediction and control of nanoemulsion droplet size.

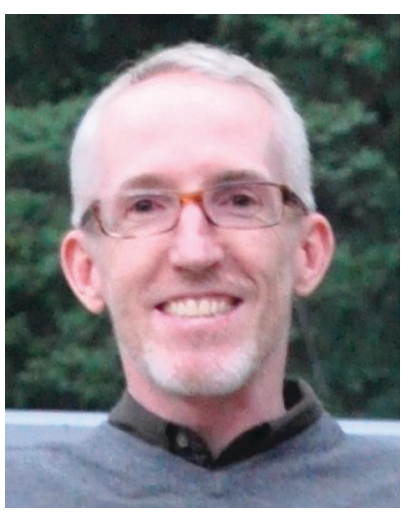

Patrick S. Doyle
Patrick S. Doyle is the Robert T. Haslam (1911) Professor of Chemical Engineering at the Massachusetts Institute of Technology. He received his PhD in Chemical Engineering in 1997 from Stanford University and then spent three years as a postdoctoral fellow at the Institute Curie in Paris. His awards include the Soft Matter Lectureship, Guggenheim Fellowship, and Pioneer of Miniaturization Lectureship. His research interests focus on soft matter, polymer physics and microfluidics. 


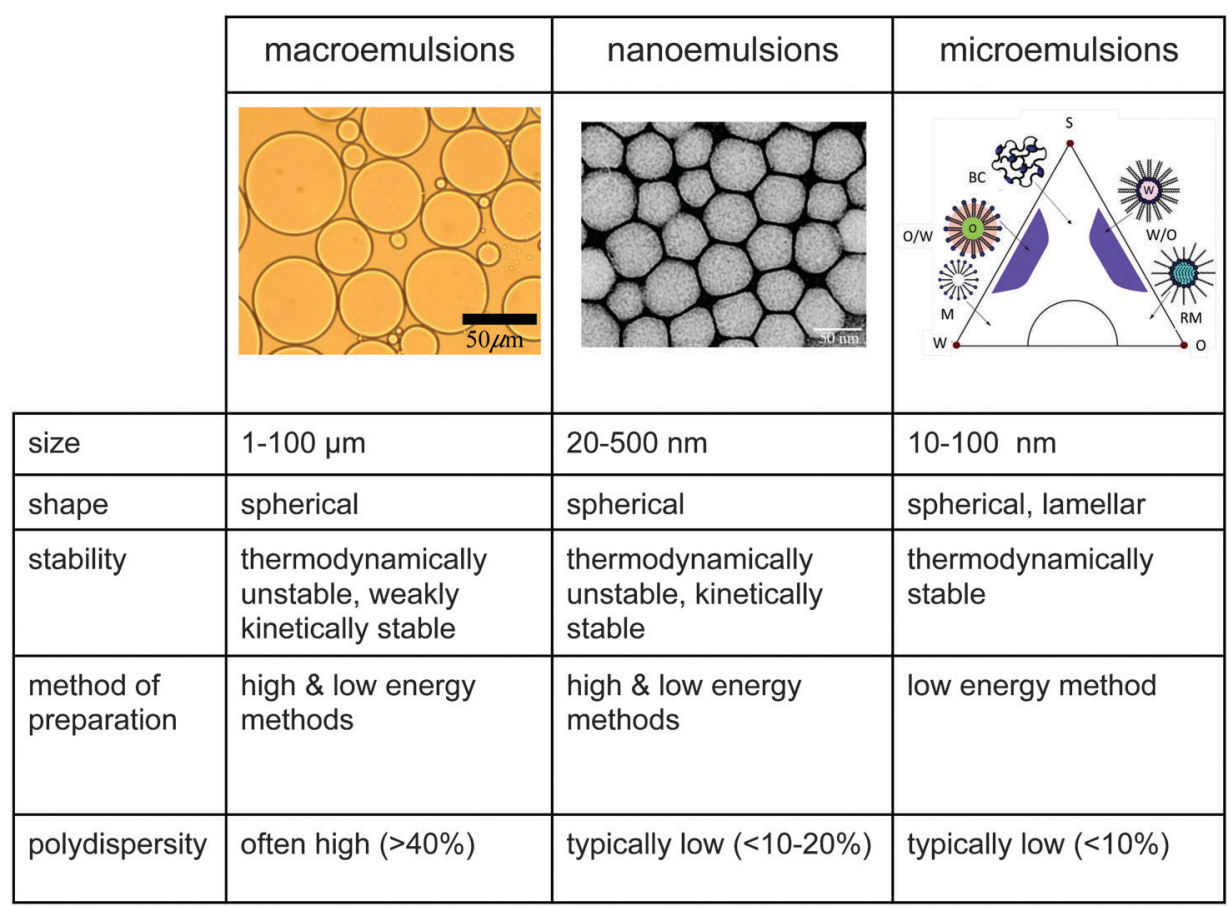

Fig. 1 Comparison of macroemulsions, nanoemulsions (also referred to as miniemulsions) and microemulsions with respect to size, shape, stability, method of preparation, and polydispersity. Nanoemulsions and microemulsions have a larger surface area per unit volume than do macroemulsions because of their size. In addition, due to a strong kinetic stability, nanoemulsions are less sensitive to physical and chemical changes. The nanoemulsion TEM image has been reprinted with permission. ${ }^{1}$ Copyright (2006) by IOP Publishing.

\subsection{Nanoemulsion preparation}

There are primarily two broad categories of techniques for the preparation of nanoemulsions: high energy methods and low energy methods. ${ }^{13-15}$ The input energy density $(\varepsilon)$ in high energy methods is on the order of $10^{8}-10^{10} \mathrm{~W} \mathrm{~kg}{ }^{-1} \cdot 82,83$ Assuming typical values of $10 \mathrm{mN} \mathrm{m}^{-1}$ for interfacial tension $(\sigma), 1 \mathrm{cP}$ for continuous and dispersed phase viscosity $\left(\mu_{\mathrm{c}}, \mu_{\mathrm{d}}\right)$, and $1000 \mathrm{~kg} \mathrm{~m}^{-3}$ for the densities of the continuous and dispersed phases $\left(\rho_{\mathrm{c}}, \rho_{\mathrm{d}}\right)$, we can obtain a rough estimate of nanoemulsion droplet size as $d \sim 100 \mathrm{~nm}$ using the correlation proposed by Gupta et al.: ${ }^{84}$

$$
\mathrm{We}=C \mathrm{Oh}^{0.4}
$$

where We is the Weber number representing the ratio of applied stress to the interfacial stress and is given by $\frac{\sqrt{\rho_{\mathrm{c}} \mu_{\mathrm{c}} \varepsilon} d}{\sigma}$; Oh is the Ohnesorge number representing the ratio of the viscocapillary breakup time scale to the Rayleigh breakup time scale and is given by $\frac{\mu_{\mathrm{d}}}{\sqrt{\rho_{\mathrm{d}} \sigma d}} ; C$ is a constant which can assumed to be around $\mathrm{O}\left(10^{-1}-10^{0}\right)$. This correlation (eqn (1)) is a modified version of the correlation proposed by Hinze ${ }^{85}$ i.e. $\mathrm{We}=\mathrm{COh}$ for $\mathrm{Oh} \ll 1$. Gupta et $a .^{84}$ argued that since typically the diameter of nanoemulsion droplets is on the order of $100 \mathrm{~nm}$, Oh $>1$. Hence, researchers derived a criterion for detachment of a filament coming out from the deformed parent drop. The correlation is useful since one is able to explain the variation of nanoemulsion droplet size for a wide variety of experimental data available in the literature, especially the data for the two most widely used higher energy methods of high pressure homogenization $(\mathrm{HPH})^{86}$ and ultrasonication. $^{2,87}$ This correlation is explained in more detail in Section 2.2.

In low energy methods, the smaller droplets are formed when the system undergoes a phase inversion in response to changes in composition or temperature, and passes through a state of low interfacial tension. These methods require significantly less input energy density $\left(\varepsilon \sim 10^{3}-10^{5} \mathrm{~W} \mathrm{~kg}^{-1}\right)$ as energy input can be achieved readily in a simple batch stirrer. ${ }^{19,83}$ Near the point of phase inversion, the system passes through a state in which the interfacial tension maybe as low as $10 \mu \mathrm{N} \mathrm{m}^{-1} \cdot{ }^{19}$ Assuming the values of $\varepsilon \sim 10^{3} \mathrm{~W} \mathrm{~kg}^{-1}, \sigma \sim 10 \mu \mathrm{N} \mathrm{m}^{-1}, 1 \mathrm{cP}$ for continuous and dispersed phase viscosity $\left(\mu_{\mathrm{c}}, \mu_{\mathrm{d}}\right)$, and $1000 \mathrm{~kg} \mathrm{~m}^{-3}$ for the densities of the continuous and dispersed phases $\left(\rho_{\mathrm{c}}, \rho_{\mathrm{d}}\right)$, one obtains $d \sim 100 \mathrm{~nm}$ from eqn (1). The two most widely used low energy methods are emulsion inversion point (EIP) ${ }^{17-19}$ and phase inversion temperature (PIT) ${ }^{16}$ EIP is sometimes referred to as phase inversion composition (PIC). Fig. 2 summarizes the preparation methods for $\mathrm{O} / \mathrm{W}$ nanoemulsions via the HPH, ultrasonication, EIP and PIT routes. W/O nanoemulsions can also be prepared in a similar fashion by reversing the dispersed and continuous phases.

As Fig. 2(a) shows, the first step in preparing an $\mathrm{O} / \mathrm{W}$ nanoemulsion through a high energy method is to prepare an $\mathrm{O} / \mathrm{W}$ macroemulsion, which is usually accomplished by mixing oil, water and surfactant in a simple batch stirrer system for a sufficient period of time; in the second step, the macroemulsion is converted into a nanoemulsion. In a homogenizer, a high 


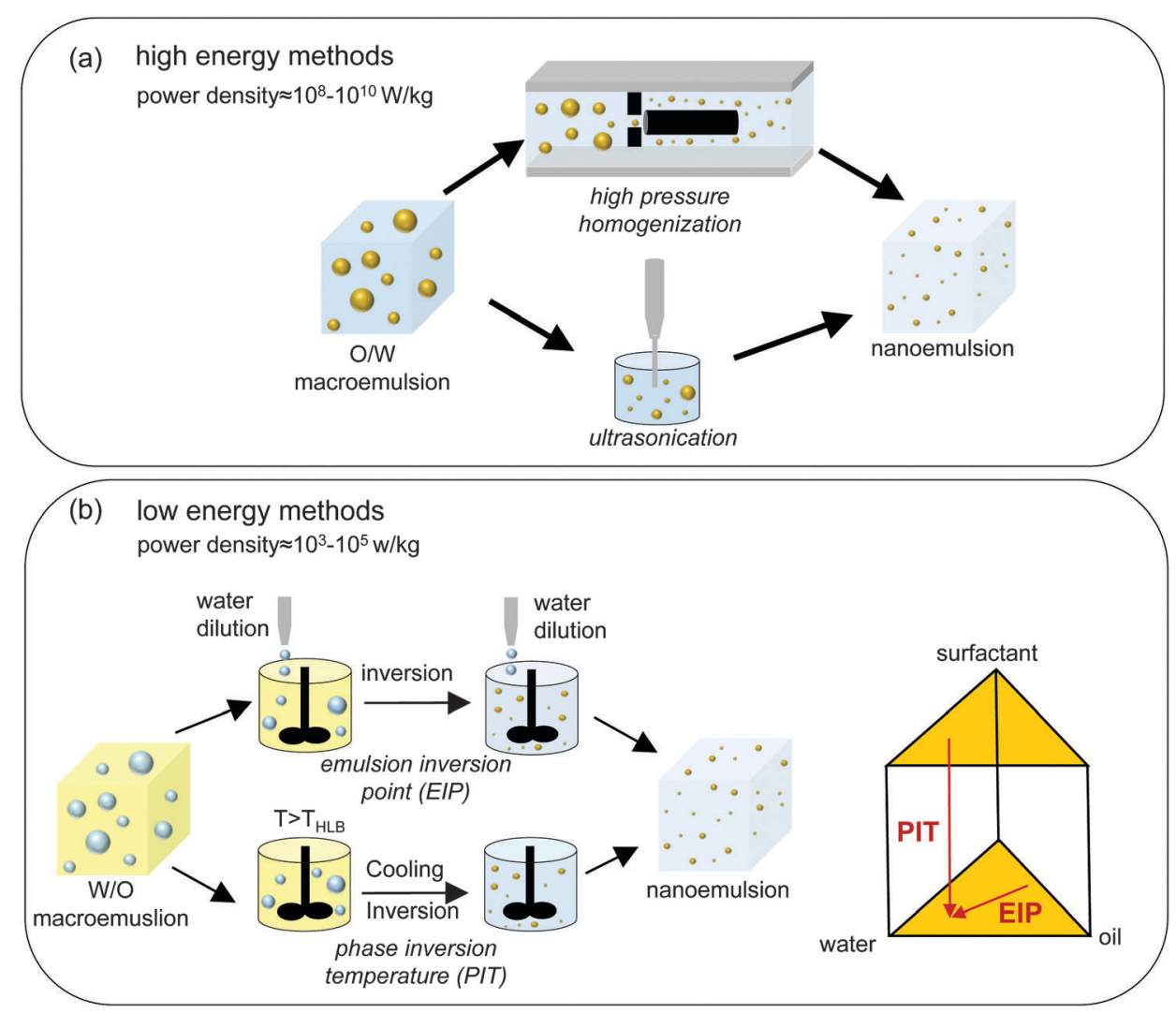

Fig. 2 Overview of high energy and low energy methods for preparing O/W nanoemulsions. (a) High energy such as high pressure homogenization (HPH) and ultrasonication break macroemulsion drops into smaller droplets. (b) Low energy methods start with W/O macroemulsions and break coarse emulsions into smaller droplets as they pass through a state of low interfacial tension during phase inversion. The emulsion inversion point (EIP) technique induces a phase inversion by water dilution whereas the Phase Inversion Temperature (PIT) approach induces a phase inversion on cooling of the mixture. To prepare W/O nanoemulsions, one can simply reverse the continuous and dispersed phases.

pressure pump pushes the macroemulsion through a narrow gap (gap height is on the order of a few microns ${ }^{88}$ ) where the large droplets break into smaller droplets as they are subjected to extreme elongational and shear stress. ${ }^{86,88}$ The homogenization process is typically repeated multiple times (referred to as the number of passes) until the droplet size becomes constant. ${ }^{89}$ In an ultrasonicator, high energy shock waves create turbulence (due to cavitation) which ruptures the droplets. As with $\mathrm{HPH}$, ultrasonication is continued until the droplet size becomes constant. $^{2}$

In contrast to high energy methods, low energy methods begin with a W/O macroemulsion which is then transformed into an $\mathrm{O} / \mathrm{W}$ nanoemulsion following changes either in composition or temperature (see Fig. 2(b)). In EIP, a W/O macroemulsion is prepared at room temperature and is then diluted slowly with water. During this dilution process, the system passes through an inversion point where the transformation from W/O to $\mathrm{O} / \mathrm{W}$ emulsion takes place. At this inversion point, the interfacial tension of the oil-water interface is very low and thus small droplets can be formed without a significant energy penalty. ${ }^{19}$ In PIT, on the other hand, the W/O macroemulsion is prepared at a temperature higher than the phase inversion temperature $\left(T_{\mathrm{HLB}}\right)$ of the mixture. When the oil-water-surfactant mixture is cooled down to room temperature, it passes through the inversion temperature at which the transformation of the mixture from a W/O to an $\mathrm{O} / \mathrm{W}$ emulsion takes place. As with EIP, the interfacial tension of the oil-water interface near the inversion point is very low, and small droplets with high specific surface area can be generated with low energy requirements. ${ }^{16}$

Other nanoemulsion preparation methods, such as bubble bursting at an oil/water interface, evaporative ripening and microfluidization, all illustrated schematically in Fig. 3, have also been demonstrated in the literature. In the bubble bursting method, gas is bubbled through an aqueous phase containing surfactant. ${ }^{20}$ Once the bubble reaches the interface (Fig. 3(a1)), the oil film between the interface and water phase drains slowly (Fig. 3(a2)), and a dimple in the water-air interface is created (Fig. 3(a3)) which nucleates and generates a spray of oil droplets in the water phase (Fig. 3(a4)). The original paper by Feng et al. ${ }^{20}$ also discusses the dependence of nanoemulsion droplet size on parameters such as oil layer thickness, bubble diameter etc.

In the evaporative ripening method proposed by Fryd and Mason $^{21}$ (Fig. 3(b)), small O/W nanoemulsion droplets can be prepared with high viscosity oils. The initial droplets produced by HPH are a mixture of volatile (low molecular weight) and nonvolatile (high molecular weight) oils; upon heating, the volatile component in these droplets evaporates and the droplets shrink and the oil phase becomes richer in the high molecular weight oil. 
(a)
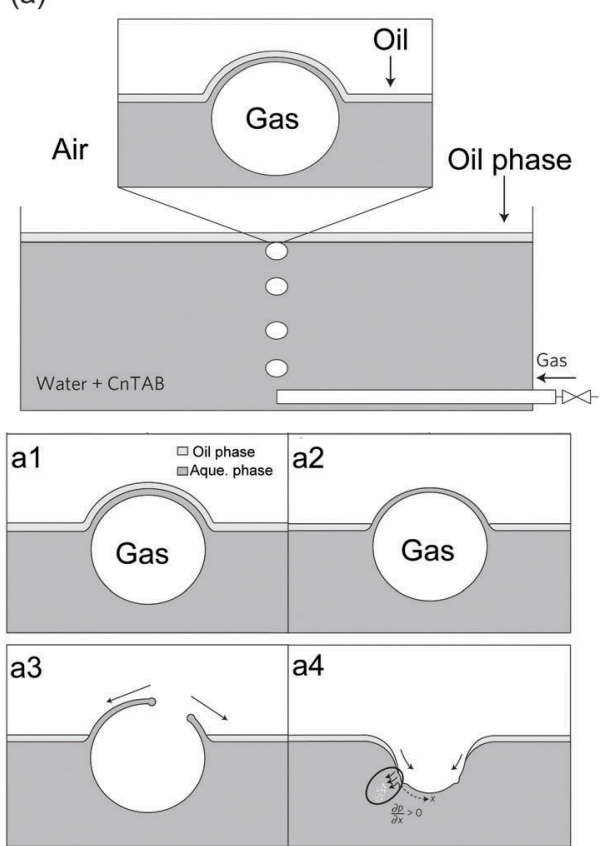

(b)

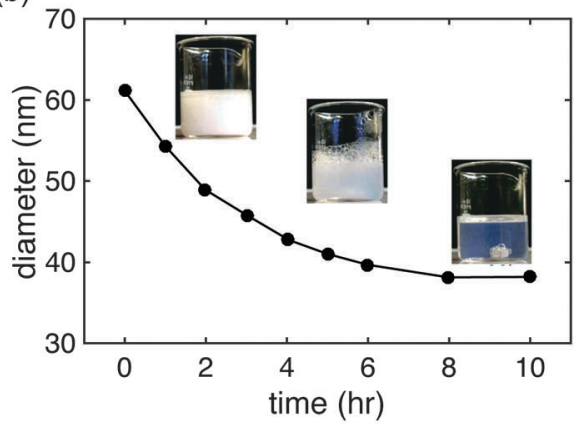

(c)

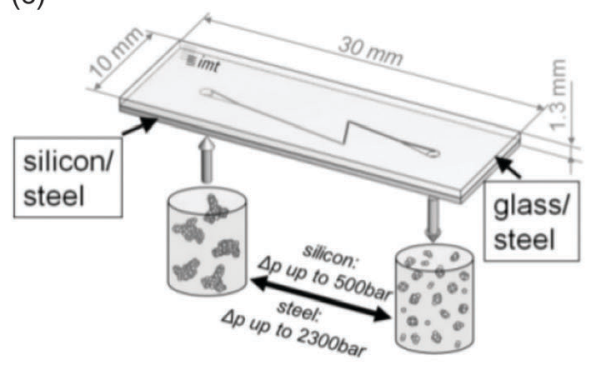

Fig. 3 Overview of novel methods for nanoemulsion preparation. (a) Nanoemulsion preparation through the bursting of a bubble at an oil-water interface. When a rising air bubble gets trapped at an oil-water interface (a1), the oil film drains out (a2) prior to bubble bursting. Once the bubble bursts (a3), a dimple forms, with the erruption of small $\left(<100 \mathrm{~nm}\right.$ ) emulsion droplets into the water phase (a4). Reprinted by permission. ${ }^{20}$ Copyright (2014) by Macmillan Publishers Limited: Nature Physics. (b) Nanoemulsion preparation through an evaporative ripening process. Nanoemulsion prepared with a mixture of a volatile and a non-volatile oil as the dispersed phase by conventional methods like HPH are heated to evaporate the volatile oil and shrink the droplets. Reprinted with permission. ${ }^{21}$ Copyright (2010) by American Chemical Society. (c) Nanoemulsion preparation through microfluidization method. In the narrower regions of microchannel, droplets experience extreme shear and rupture. This concept is similar to that of a high pressure homogenizer. Copyright (2011) by Wiley-VCH Verlag GmbH \& Co. KGaA, Weinheim.

Microfluidization, for the preparation of nanoemulsions (Fig. 3(c)) is similar to high pressure homogenization ( $\mathrm{HPH})$, and the terms are sometimes used interchangeably. ${ }^{90}$ The microfluidizer has a well-defined axially-varying microchannel geometry through which macroemulsions are pumped. In the narrow throats spaced along the length of the microchannel, the drops experience high shear rates and break $^{90}$ to form smaller droplets. As in $\mathrm{HPH}$, microfluidization requires multiple passes for the droplet to attain a constant final size.

\subsection{Droplet size control}

An important aspect of nanoemulsion preparation through the above-mentioned techniques is control of the final droplet size $(d)$, which can be influenced by residence time, relative viscosity $\left(\mu_{\mathrm{d}} / \mu_{\mathrm{c}}\right)$, surfactant concentration ([S]) and surfactant length $(L)$. In their recent work, Gupta et al. ${ }^{84}$ proposed a new scaling relation which builds upon the classical expression proposed by Hinze, ${ }^{85} \mathrm{We}_{\text {crit }} \sim C(1+f(\mathrm{Oh}))$. Hinze ${ }^{85}$ argued that We and $\mathrm{Oh}$ are the two most important dimensionless numbers that are important for understanding droplet breakup. However, Hinze only discussed the limiting case of Oh $\ll 1$ where $f(\mathrm{Oh})$ vanishes from the expression. Modern nanoemulsion literature has relied heavily on the correlation developed by Hinze or its modifications. ${ }^{1,2,87,89}$ Gupta et al. ${ }^{84}$ argued, however, that for nanoemulsions, Oh $\sim$ 1-100 due to the small droplet sizes, and proposed the physical model of droplet breakup shown in Fig. 4(a). The authors argue that the important dimensionless quantity is the Weber number of the filament trying to detach from the droplet, and showed that $\mathrm{We}_{\text {crit, }}$ will reduce to the parent droplet Weber number (We) in the case of $\mathrm{Oh} \ll 1$, as predicted by Hinze. ${ }^{85}$ However, in the other limit of $\mathrm{Oh} \gg 1$, $\mathrm{We}_{\text {crit,a }}$ is dependent on Oh such that $\mathrm{We}_{\text {crit, } \mathrm{a}} \sim C$ is equivalent to We $\sim \mathrm{COh}^{0.4}$. The authors further argued that We should be carefully defined because though the Re of the continuous phase will suggest that the flow is turbulent in bulk, $\mathrm{Re}_{\mathrm{d}}$ will be of the order of unity, suggesting that the flow is viscous around droplets. In other words, the droplets will be smaller than the smallest eddy size i.e. Kolmogorov's eddy size as predicted by the classical turbulence theory (Fig. 4(b)). For instance, Kolmogorov's length scale, $\lambda \sim\left(\frac{\varepsilon \rho_{\mathrm{c}}^{3}}{\mu_{\mathrm{c}}^{3}}\right)^{0.25}$ is about $300 \mathrm{~nm}$ for typical high energy methods. Hence, We should be based on the stress inside a Kolmogorov's eddy or $\mathrm{We} \sim \frac{\sqrt{\mu_{\mathrm{c}} \rho_{\mathrm{c}} \varepsilon} \sigma}{d}$. Gupta et al. showed that the proposed scaling is able to validate a large range of experimental data for both homogenizer and ultrasonicator (Fig. 4(c) and (d)) spanning a large range of Oh. The experimental data clearly shows an increase in droplet size for higher values of $\mu_{\mathrm{d}}$ which is then manifested in a higher value of $\mathrm{Oh}$.

The results of Delmas et al. ${ }^{2}$ shown in Fig. 5(a) give the transient evolution of nanoemulsion droplet size during ultrasonication as affected by surfactant concentration and length. 
(a)

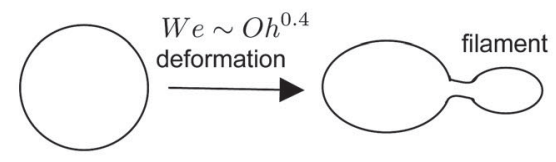

(c)

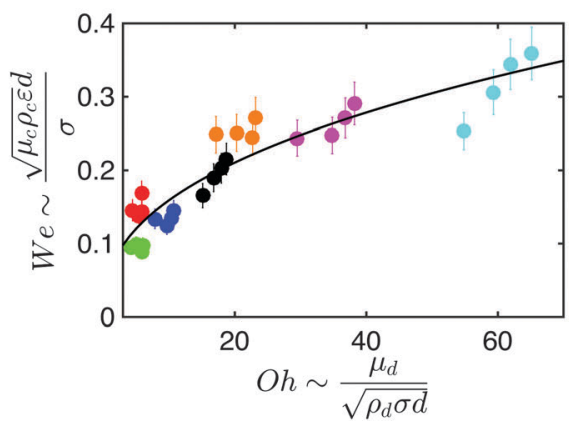

(b)

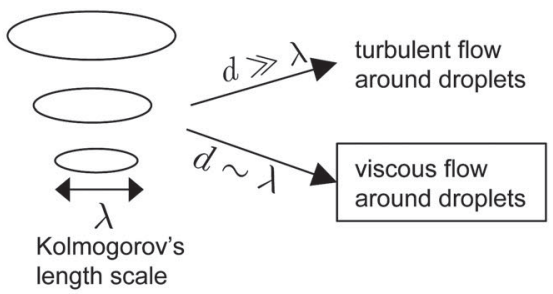

(d)

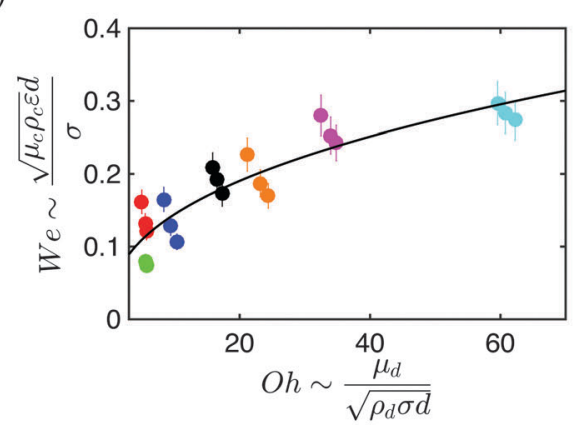

Fig. 4 Physical model for nanoemulsion formation. (a) Typical droplet deformation process in which a droplet deforms such that a filament starts to grow and eventually breaks away. Gupta et al. ${ }^{84}$ showed below a critical Weber number, $W_{\text {crita,a, }}$ the filament is unable to detach from the droplet. The equivalent relation expressed in the dimensions of parent droplet is $\mathrm{We} \sim \mathrm{Oh}^{0.4}$. (b) The flow around nanoemulsions is viscous whereas the flow in the continuous phase is turbulent. This happens because the droplet size of nanoemulsions (d) is smaller than the smallest eddy given by the Kolmogorov's length scale $(\lambda)$. (c and $d$ ) Validation of the proposed scaling for a wide range of We and Oh. The different colors represent different systems and the solid line represents the theoretical prediction.

The droplet size becomes roughly constant upon increase in ultrasonication time. A similar dependence of droplet size in HPH was reported by Mason et al.,${ }^{89}$ and our group, ${ }^{81,84}$ where the droplet size was observed to approach a constant value with an increased number of passes. In all cases, the droplet size decayed exponentially with increasing ultrasonication time or, in the case of HPH, with the number of passes, a clear sign that droplet breakage dominates over coalescence during nanoemulsion formation. ${ }^{84,91}$ As Fig. 5(a) shows, the final droplet size attained after 20 minutes processing time decreases with an increase in surfactant concentration, attributed to the decreasing interfacial tension, which makes it easier to break the droplets (see eqn (1)). ${ }^{2}$ The increase in droplet size due to increased surfactant length has also been attributed to a change in interfacial tension. ${ }^{2}$ Similar results have been obtained by Mao et al. ${ }^{63,64}$ Fig. 5 (b) shows the work by Wooster et al. ${ }^{87}$ where the effect of the relative viscosity $\left(\mu_{\mathrm{d}} / \mu_{\mathrm{c}}\right.$, the ratio of the viscosity of the dispersed phase relative to that of the continuous phase) on the droplet size was observed. They prepared the $\mathrm{O} / \mathrm{W}$ nanoemulsions by microfluidization and systematically added polyethylene glycol (PEG) to increase the continuous phase viscosity. As Fig. 5(b) shows, the size of the nanoemulsion droplets increased with an increase in the viscosity ratio. This effect can be explained by eqn (1) since the We number increases when one increases $\mu_{\mathrm{c}}$ whereas Oh number remains constant. However, increase in the length of the surfactant suppressed the effect of relative viscosity. This effect has not been captured in the scaling relation proposed by Gupta $e t$ al. because the analysis currently assumes that the surfactant length is much smaller than the diameter of the drops. ${ }^{84}$ Qian and McClements ${ }^{8}$ also observed similar results. In high energy methods, few studies have also focused on understanding the effect of homogenizer geometry on the droplet size distribution..$^{90,92,93}$ These studies concluded that the droplet size distribution can be better controlled by tuning the geometry of the homogenizer/microfluidizer.

There also exists a vast literature on controlling the size of nanoemulsion droplets prepared through low energy methods. Fig. 5(c) shows the result of work by Pey et al. ${ }^{94}$ where researchers prepared nanoemulsions through EIP and systematically varied a large number of parameters. As Fig. 5(c) shows, the ratio of oil to surfactant concentration $([\mathrm{O}] /[\mathrm{S}])$, and the ratio of additive to surfactant concentration $([\mathrm{A}] /[\mathrm{S}])$, both affect the droplet size. Several other studies report the dependence of nanoemulsion droplet size on a large number of parameters. ${ }^{17-19,25,95}$ However, the results are mainly empirical, and little theoretical understanding is presented to explain these variations. Another section of literature focused on discerning the mechanism of formation of nanoemulsions prepared through low energy methods. ${ }^{16,24,26,96}$ These studies concluded that nanoemulsions form when (i) the system passes through the region of bicontinuous microemulsions upon dilution in EIP, or (ii) the starting phase is a bicontinuous microemulsion in PIT. Fig. 5(d) shows the work of Rao and McClements ${ }^{9}$ who compared nanoemulsions prepared through EIP and HPH. As Fig. 5(d) indicates, HPH produces small droplets at a significantly lower surfactant concentration than does EIP. This is one of the downsides of nanoemulsions prepared through low energy methods. This behavior could be due to the absence of a low interfacial tension region for a low surfactant concentration. Some studies ${ }^{71,97-100}$ have 
(a)

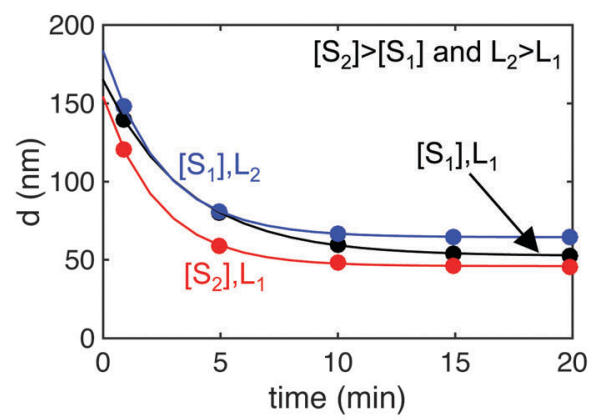

(b)

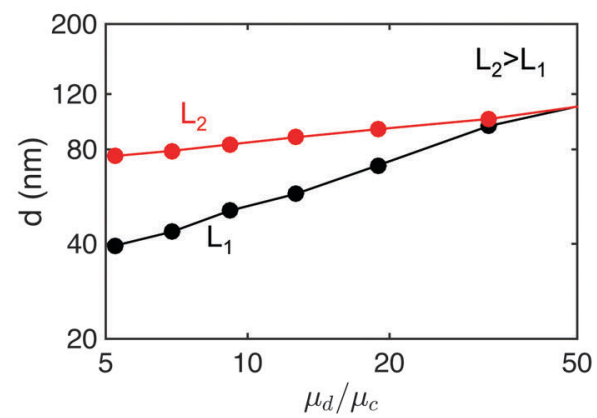

(c)

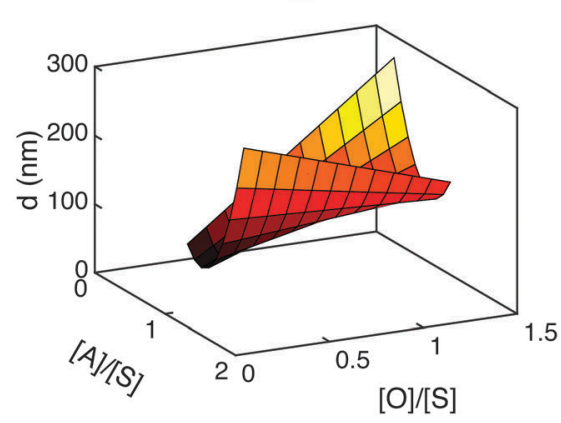

(d)

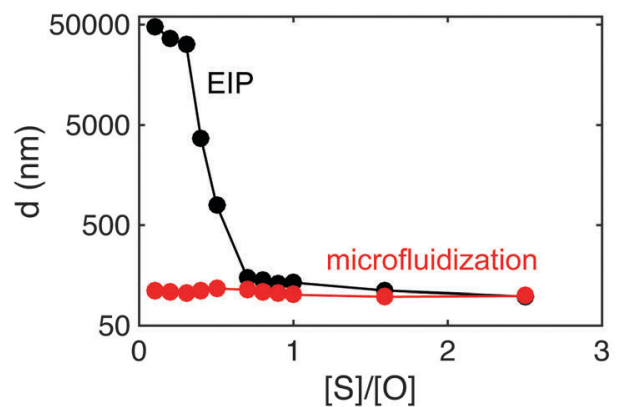

Fig. 5 Results from the literature on the control of the droplet size in high and low energy methods. (a) Droplet size of nanoemulsions prepared by ultrasonication for different ultrasonication time, surfactant concentrations ([S1] and [S2]) and surfactant lengths (L1 and L2). Results indicate that breakage mechanism dominates over coalescence as droplet size decays exponentially with ultrasonication time. Also, due to changes in interfacial tension, droplet size decreases with (i) an increase in surfactant concentration and (ii) a decrease in surfactant length. Data from Delmas et al. ${ }^{2}$ (b) Nanoemulsion prepared by ultrasonication for different relative viscosity ratio and surfactant lengths. The diameter increases with an increase in viscosity ratio but with an increase in the surfactant length, the effect of relative viscosity decreases. Data from Wooster et al. ${ }^{87}$ (c) Nanoemulsions prepared by EIP with different oil, surfactant and additive concentrations. Results show that size depends strongly on the composition of the nanoemulsions. Data from Pey et al. ${ }^{94}$ (d) Effect of surfactant-to-oil concentration on droplet size of emulsions prepared by EIP and microfluidization. Results show that relatively less surfactant is required in high energy methods to produce droplets in the nano-size range. Data from Ostertag et al. ${ }^{5}$

also compared the droplet size of nanoemulsions prepared through ultrasonication and homogenization/microfluidization, and concluded that for the same power density input, the droplet size obtained is similar.

There are several unanswered questions that need to be addressed for understanding nanoemulsion formation in greater depth. For instance, what are the process parameters and material properties that control the droplet size distribution? What is the role of the kinetics of surfactant diffusion and adsorption during the rapid droplet formation? Experimental as well as modeling studies should be performed in the future to answer the above questions. Direct visualization of nanoemulsion droplets could be investigated through advanced microscopy techniques ${ }^{101}$ which would lead to a better understanding of intradroplet interactions. Future efforts should also focus on ways to scale up the low energy methods through millifluidics and microfluidics, akin to recent approaches for larger-sized emulsions. ${ }^{102}$

\section{Stability}

This section describes the fundamentals behind emulsion destabilization mechanisms and systematically presents the reasons for robust stability of nanoemulsions. This section also details the research conducted on understanding and controlling nanoemulsion stability.

\subsection{Destabilization mechanisms}

Nanoemulsions are kinetically stable and given sufficient time, will separate into different phases. Fig. 6 summarizes the different destabilization mechanisms of nanoemulsions namely flocculation, coalescence, Ostwald ripening and creaming/ sedimentation.

In flocculation, droplets come closer to each other because of attractive interactions and move as a single entity. In contrast, during coalescence, the droplets merge into each other to become a bigger drop ${ }^{103}$ (Fig. 6). The DLVO theory predicts that when the repulsive maximum of the droplet-droplet interaction potential is low $\left(\sim 0 k_{\mathrm{B}} T\right)$, droplets come close to each other and fall into the primary minimum i.e., the irreversibly flocculated state. During this process, when the droplets come into 'primary' contact, they tend to coalesce. ${ }^{103}$ Therefore, it is hard to distinguish between flocculation and coalescence in emulsions. Typically, due to the adsorbed layer of emulsifier on the droplet, steric interactions increase the repulsive maximum which in turn stabilize the emulsions against flocculation and coalescence. The steric stabilization is stronger in nanoemulsions 


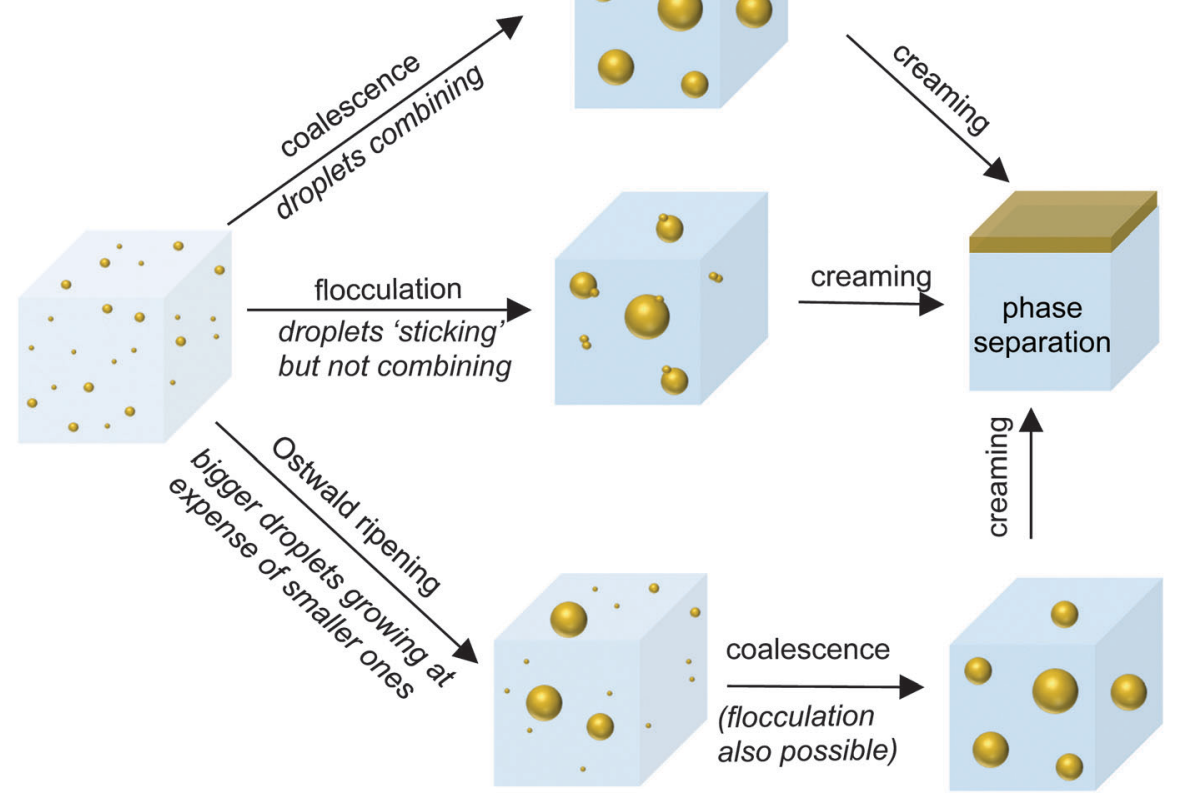

Fig. 6 Schematic of various nanoemulsion destabilization mechanisms. Though nanoemulsions can be destabilized through any of the possible routes, initial growth of droplets generally occurs through Ostwald ripening. However, coalescence and flocculation become more important as the droplet size increases.

as the thickness of the adsorbed emulsifier layer $(\sim 10 \mathrm{~nm})$ is comparable to the droplet size. ${ }^{13}$

Ostwald ripening occurs due to the difference in chemical potential of solute within droplets of different sizes. Due to Laplace pressure $\left(P_{\mathrm{L}}=\frac{4 \sigma}{d}\right)$, the chemical potential of the dispersed phase is higher in smaller droplets than in larger ones, providing the driving force for mass transfer from the smaller to the larger droplets. Thus the smaller droplets become smaller and the larger droplets grow (Fig. 6). As the mass transfer occurs when the dispersed phase molecule travels through the continuous phase, solubility of the dispersed phase in the continuous phase is a critical factor affecting Ostwald ripening rate. $^{1}$ In a classical paper, Lifshitz and Slyozov $^{104}$ derived the following rate equation for Ostwald ripening from first principles:

$$
\bar{d}^{3}=\bar{d}_{0}^{3}+\frac{64 \sigma C_{\infty} \nu^{3} \mathscr{D}}{9 R T} t
$$

where, $\bar{d}_{0}$ is the initial number average diameter, $\sigma$ is the interfacial tension, $C_{\infty}$ is the solubility of the dispersed phase in the continuous phase, $\nu$ is the molar volume of the dispersed phase, $\mathscr{D}$ is the diffusivity of the dispersed phase in the continuous phase, $R$ is the ideal gas constant and $T$ is the temperature of the system. The derivative of eqn (2) gives the following expression:

$$
\frac{\mathrm{d}}{\mathrm{d} t}(\bar{d}) \sim \frac{\omega_{\mathrm{O}}}{\bar{d}^{2}}
$$

where $\omega_{\mathrm{O}}$ is the Ostwald ripening rate constant and has the value $\frac{64 \sigma C_{\infty} \nu^{3} \mathscr{D}}{27 R_{\mathrm{g}} T}$. Eqn (3) shows that the rate of Ostwald ripening scales as $1 / \bar{d}^{2}$. Hence, this destabilization mechanism is more prevalent in nanoemulsions as compared to macroemulsions. As discussed later, researchers have also observed this experimentally. ${ }^{2,16}$ Delmas et $a .^{2}$ reported that other destabilization mechanisms like flocculation and coalescence become more important at later times. ${ }^{2}$

As Fig. 6 shows, in creaming, droplets rise due to buoyancy, eventually leading to phase separation (similarly, in sedimentation, droplets settle down). Creaming occurs when the buoyant force dominates over the thermal fluctuations. The ratio of viscous stress to thermal stress is given by the Peclet number, Pe $\sim \mu_{\mathrm{c}} U d^{2} / k_{\mathrm{B}} T$, where $U$ represents the velocity of the rising droplet and $k_{\mathrm{B}}$ represents the Boltzmann constant. ${ }^{105}$ For a typical droplet of $d \sim 1 \mu \mathrm{m}$ in an aqueous system at ambient temperature, terminal velocity $U \sim 1 \mu \mathrm{m} \mathrm{s}^{-1}$ and hence, $\mathrm{Pe} \sim 1$. However, for a nanoemulsion of $d \sim 100 \mathrm{~nm}, U \sim 10 \mathrm{~nm} \mathrm{~s}^{-1}$ $\left(U \propto d^{2}\right)$ and hence, Pe $\sim 10^{-4}$. Therefore, creaming is insignificant in nanoemulsions until the droplet size increases to the order of a few microns due to Ostwald ripening, flocculation and coalescence.

\subsection{Controlling stability of nanoemulsions}

Fig. 7 shows the summary of the work done on controlling nanoemulsion destabilization. Fig. 7(a) lists various parameters that affect the destabilization rate of nanoemulsions. 
(a)

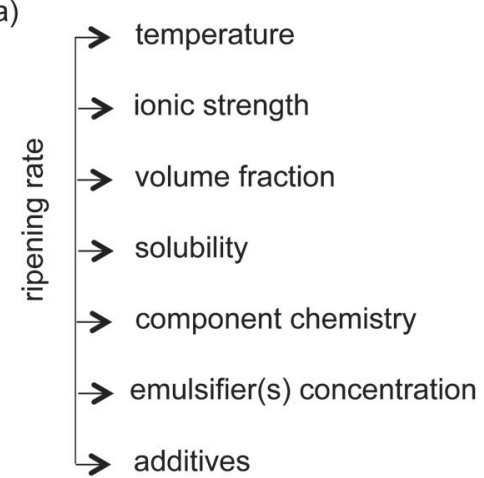

(c)

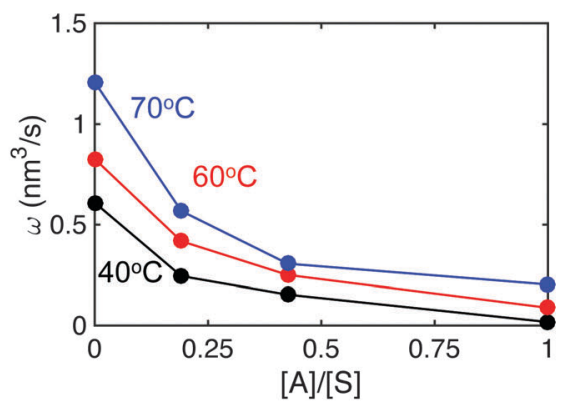

(b)

low energy method

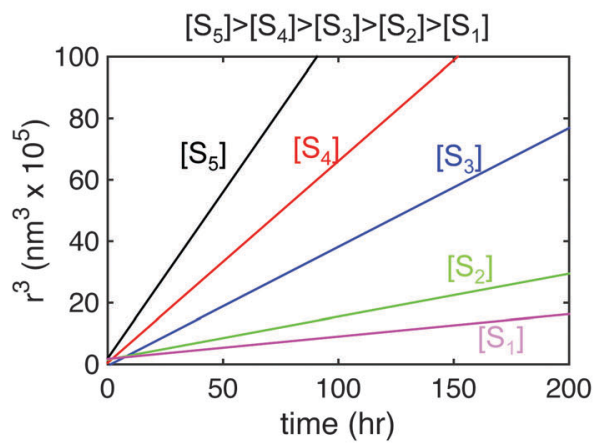

(d)

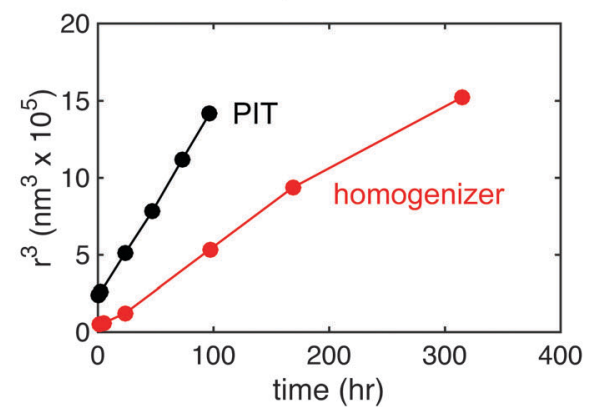

Fig. 7 Literature results on the stability of nanoemulsions. (a) List of various factors that control the stability of nanoemulsions (b) changes in stability of nanoemulsions (prepared using PIT) with changes in surfactant concentration. The stability decreases with an increase in surfactant concentration. Data from Izquierdo et al. ${ }^{16}$ (c) Effect of changes in concentration of an insoluble emulsifier ([A]) on the stability of nanoemulsions prepared using ultrasonication. The stability improves with increase in $[\mathrm{A}]$, much like in the trapped species method. Also, the stability decreases at higher temperature due to changes in solubility and diffusivity. Data from Delmas et al. ${ }^{2}$ (d) Comparison of stability profile of nanoemulsions prepared through PIT and HPH. Nanoemulsions are more stable when prepared by HPH due to lower polydispersity. Data from Tadros et al. ${ }^{106}$

Delmas et $a .^{2}$ showed that the Ostwald ripening rate $\omega_{\mathrm{o}}$ follows an Arrhenius behavior with $\omega_{\mathrm{o}} \propto \exp \left(-E / k_{\mathrm{B}} T\right)$. This happens because both solubility and diffusivity are temperature dependent. The ionic strength of the continuous phase significantly affects the repulsive barrier between the two droplets. ${ }^{105,107}$ Increase in the ionic strength of the continuous phase significantly reduces the Debye screening length and hence a lower repulsive barrier is observed. Thus, the emulsions have a much higher probability of flocculating/coalescing. Polydispersity affects the Ostwald ripening rate significantly as higher polydispersity represents a higher difference in chemical potential between droplets. As mentioned, solubility of the dispersed phase in the continuous phase affects the Ostwald ripening because a higher solubility makes it easier for dispersed phase molecules to travel through the continuous phase. Other listed parameters such as component chemistry, emulsifier concentration, and additive concentration alter destabilization rates due to changes in properties like interfacial tension $(\sigma)$, droplet elasticity, and interaction potential between droplets.

Fig. 7(b) shows the work by Izquierdo et al. ${ }^{16}$ who measured the droplet size evolution of nanoemulsions prepared through PIT with different surfactant concentrations. As Fig. 7(b) shows, the plot of $r^{3}$ vs. $t$ is a straight line, indicating that Ostwald ripening is the dominant mechanism. One observes that the rate of destabilization increases with an increase in the surfactant concentration. It has been argued that this effect is attributed to an enhanced diffusion of oil due to micelle formation and lowering of Gibbs elasticity. ${ }^{16}$ Other researchers have also studied the effect of oil and surfactant concentration on nanoemulsion stability. ${ }^{24,26-29,94,108}$ The results do not show a consistent trend because the inter-droplet interactions are different for different systems.

Delmas et $a l^{2}{ }^{2}$ and Wooster et $a l^{87}$ have tested the trapped species method in which an insoluble additive was introduced in the dispersed phase of nanoemulsions prepared by ultrasonication. This approach relies on the effect of dissolved species on the osmotic pressure $\left(P_{\mathrm{O}}\right)$. If the small droplets were to shrink and the large ones to grow because of Ostwald ripening, then the concentration of the trapped species in the small droplets would increase, and so would the osmotic pressure; the concentration in the large droplets would decrease, with a concomitant decrease in osmotic pressure. The driving force for transport from the small to the large droplets is $\Delta\left(P_{\mathrm{L}}-P_{\mathrm{O}}\right)$, so the osmotic pressure effect counteracts the Laplace pressure driving force. $\Delta P_{\mathrm{L}} \sim\left(\frac{1}{d_{\text {small }}}-\frac{1}{d_{\text {large }}}\right)$ increases as Ostwald ripening progresses $\left(d_{\text {small }}\right.$ decreases, $d_{\text {large }}$ increases), as does $\Delta P_{\mathrm{O}} \sim\left(\frac{1}{d_{\text {small }^{3}}}-\frac{1}{d_{\text {large }^{3}}}\right)$, but at a faster rate. At some point the two cancel each other out and the driving force 
for transport becomes negligible. Researchers have observed the suppression of Ostwald ripening rates for nanoemulsions using the trapped species method. ${ }^{2,87}$ In a variation of this approach, instead of adding an additive to the dispersed phase, an insoluble emulsifier is introduced into the continuous phase. Fig. 7(c) shows the work of Delmas et $a .^{2}{ }^{2}$ where $\omega_{\mathrm{o}}$ decreases upon an increase in the concentration of an insoluble emulsifier ([A]). This effect is similar to the trapped species method as the insoluble emulsifier introduces an additional penalty to the chemical potential description. However, this method was less effective for later times as the addition of an insoluble emulsifier, increased the flocculation/coalescence rate due to reduction in steric repulsion between droplets. Fig. 7(c) also shows an increase in the ripening rate with an increase in the temperature due to change in dispersed phase solubility and diffusivity. Other investigators have also reported similar dependence of stability on temperature. ${ }^{17,26}$ Fig. 7(d) which compares the stability of nanoemulsions prepared through PIT and HPH, shows that the rate of destabilization is lower in nanoemulsions prepared by HPH relative to the ones prepared by PIT. This effect has been attributed to the higher polydispersity of nanoemulsions prepared through PIT.

The emulsifier plays a critical role in both the formation and stability of a nanoemulsion. The emulsifier choice (be it surfactant, protein or lipid) is often limited by commercial availability. Custom synthesized polymeric emulsifiers will offer more flexibility with tailored size, hydrophobicity, light (e.g. azobenzene modified) or salt (e.g. zwitterionic) responsive groups, multiblock polymers, and topology. This will enable the preparation of nanoemulsions with ability to stabilize/destabilize in response to physical or chemical changes. These polymer-stabilized nanoemulsions would be also an interesting model system since the thickness of the polymeric emulsifier can be comparable to the droplet size - a relatively unexplored regime in soft-colloidal physics.

\section{Properties and applications}

Nanoemulsions have unique properties such as small droplet size, exceptional stability, transparent appearance and tunable rheology. These properties make nanoemulsions an attractive candidate for applications in the food, cosmetic, pharmaceutical industries and in drug delivery applications (Fig. 8). Furthermore, they can serve as the building blocks for designer advanced materials with unique properties. In this section, we review the literature on nanoemulsion properties and applications.

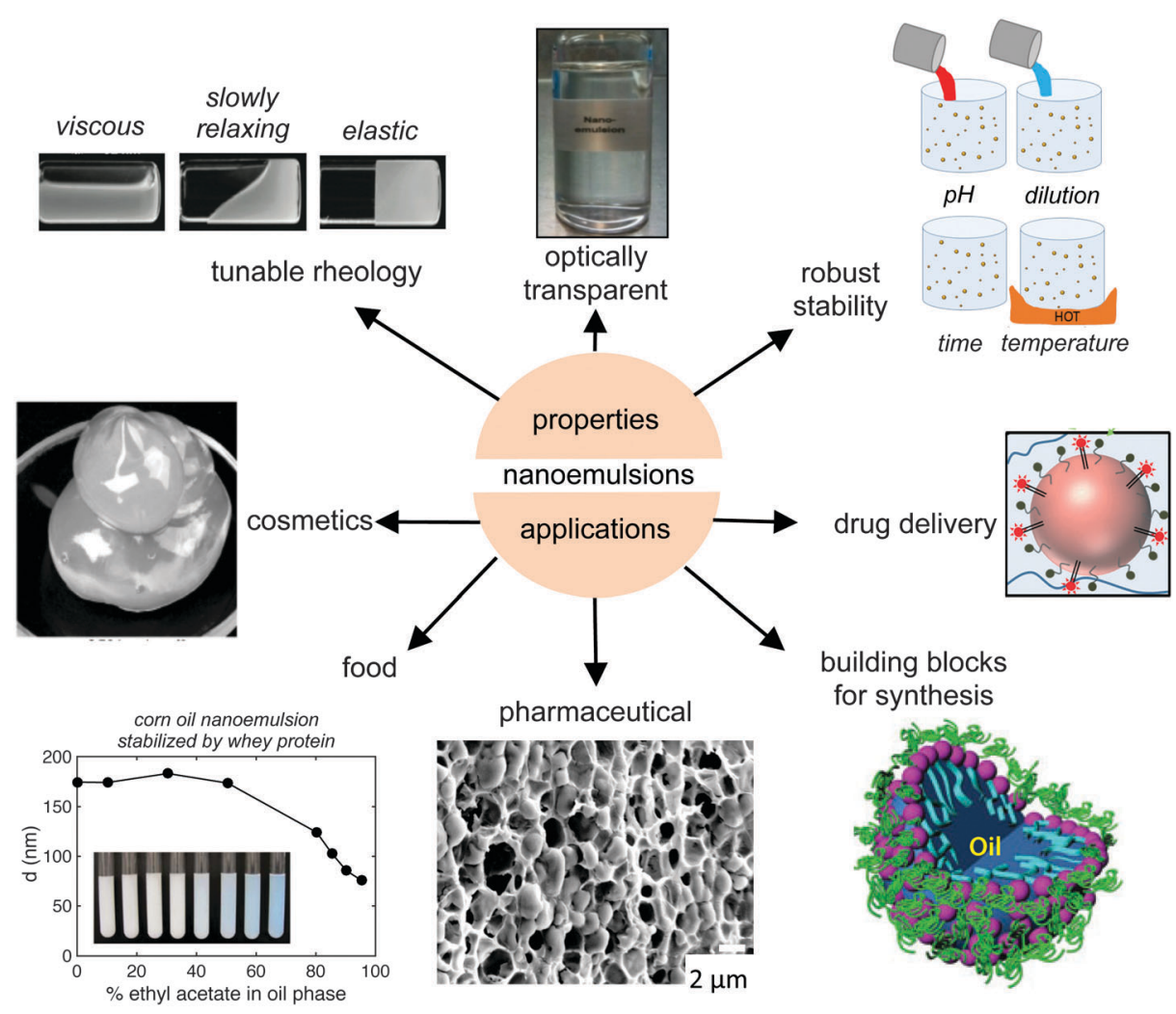

Fig. 8 Schematic of various properties and applications of nanoemulsions. Nanoemulsions have attractive properties such as small size, low polydispersity, kinetic stability and tunable rheology. Nanoemulsions find applications in cosmetics, the food and pharmaceutical industry, ${ }^{76}$ drug delivery, and as building blocks for material synthesis. ${ }^{109}$ Image of tunable rheology adapted with permission. ${ }^{15}$ Copyright (2012) by Annual Reviews. Image of cosmetics has been adapted with permission. ${ }^{74}$ Copyright (2004) by Elsevier. Image of food adapted with permission. ${ }^{3}$ Copyright (2011) by Royal Society of Chemistry. Image of pharamaceuticals adapted with permission. ${ }^{76}$ Copyright (2014) by American Chemical Society. Image of building blocks for synthesis adapted with permission. ${ }^{15}$ Copyright (2012) by Annual Reviews. Image of drug delivery adapted with permission. ${ }^{80}$ Copyright (2014) by Royal Society of Chemistry. 


\subsection{Droplet size and stability}

Nanoemulsions have droplets with diameter on the order of $100 \mathrm{~nm}$. As the droplet size is significantly smaller than the wavelength of visible light, nanoemulsions are often transparent in appearance (Fig. 8). However, by controlling the droplet size of nanoemulsions, one can easily tune the appearance of nanoemulsions to range from transparent to milky white. ${ }^{2}$ As discussed in Section 3.1, nanoemulsions can also be tuned to have robust stability with shelf life ranging from months to years. They have an added advantage of being relatively less sensitivity towards dilution, temperature and $\mathrm{pH}$ changes than are microemulsions. These properties make nanoemulsions attractive in various industries, including in the food and cosmetic sectors.

\subsection{Tunable rheology}

The rheological properties of nanoemulsions can be tuned by controlling the dispersed phase volume fraction and droplet size, and by the addition of salt and depletion agents. Nanoemulsions are particularly interesting from a rheological point of view as they can exhibit significantly stronger elasticity than macroemulsions as the elasticity is roughly on the order of the Laplace pressure of an undeformed droplet i.e. $P_{\mathrm{L}} \sim \frac{\sigma}{d}$. The tuning of the rheology of an emulsion often dictates the consumer's perception of the product in the cosmetic industry (Fig. 8). ${ }^{74}$

Wilking et al. ${ }^{110}$ showed that one can tune the flow behavior of the nanoemulsions to be from a flowing fluid to a slowly relaxing fluid or a gel-like system by changing the number of passes in a $\mathrm{HPH}$ (Fig. 8). This irreversible flow-induced elastification happens due to excess droplet rupture leading to jamming of the nanoemulsion structure. One can also tune the rheological response of nanoemulsions by adding salt or a depletion agent which leads to gelling of droplets. Another way of tuning rheological behavior is to add polymers that can physically associate among themselves or with nanoemulsion droplets. ${ }^{15,74}$ For instance, our group ${ }^{81}$ added a polymer gelator with hydrophobic end groups to create thermoreversible gels. This reversible phenomenon is claimed to be due to changes in hydrophobic interactions between the polymer and the droplets with temperature. At temperatures higher than the gelling temperature $\left(T_{\mathrm{g}}\right)$, the polymer with two hydrophobic end groups bridges the nanoemulsion droplets, creating a percolated network. When the temperature is brought down below $T_{\mathrm{g}}$, the hydrophobic groups detach from the surface of nanoemulsion droplets and the system returns to its initial transparent and fluid-like state. The study showed that the relative value of the interactions length (radius of gyration of the bridging polymer) to the droplet size plays an important role. Strong gels will form when the length scale of the attractive interaction is comparable to the droplet size. This explains why macroemulsions form pastes with a low elastic modulus. More recent studies have highlighted that the nanoemulsions can undergo arrested spinodal decomposition ${ }^{111}$ and that the gelation is sensitive to the rate at which the attractive interactions (via temperature) is increased. ${ }^{112}$ Future studies should investigate the rheological behavior of nanoemulsionbased gels in greater depth. Since the relative value of interaction length to droplet size play an important role, nanoemulsions with bi-modal or tri-modal distributions can be used to create gels with rich rheology. Further studies on rupture (yielding) of nanoemulsion-based gels ${ }^{113}$ could provide insights into the association and disassociation mechanism of the bridging gelator, and perhaps healing behavior of the system.

\subsection{Nanoemulsions in drug delivery}

Fig. 9(a) shows the various modes of drug administration in the human body. Nanoemulsions have been used in most forms of drug delivery, namely topical, ocular, intravenous, internasal and oral delivery. These applications leverage the lyphophilic nature of nanoemulsions to solvate water-insoluble drugs; and tunable charge and rheology of nanoemulsions to formulate aqueous solutions that can be easily delivered to patient.

Though skin protects us from the external environment, it also acts as a transport barrier against administration of drugs through the skin. Topical medication formulated using nanoemulsions can provide unique advantages as the dispersed phase of $\mathrm{O} / \mathrm{W}$ nanoemulsions enables enhanced solubility of lipophilic drugs in the oil phase and the continuous phase provides a mild, skin-friendly environment that can dissolve biopolymers such as alginate for adjusting the formulation rheology, appearance and texture. A considerable number of studies focused on using nanoemulsions for topical drug delivery. ${ }^{30-47,60}$ Few of the above-mentioned studies included permeation tests to evaluate the effectiveness of topical delivery. ${ }^{40,45-47,60}$ Some studies claim that owing to the relatively small size and low $\zeta$-potential of nanoemulsion formulations, hydrophobic drugs are delivered more efficiently than are suspensions of these drugs. ${ }^{46,47}$ Mou et al. prepared hydrogel thickened nanoemulsions that had higher permeation rates as compared to conventional hydrogels. ${ }^{36}$ Researchers have also explored the use of nanoemulsions in other modes of drug delivery such as ocular, ${ }^{48-50}$ intravenous, ${ }^{51,55}$ intranasal $^{52,53}$ and oral delivery. ${ }^{54,56-62,116}$ In these studies, a pharmaceutical drug is dissolved in the dispersed phase, and formulations are tested for bioavailability and delivery efficiency in environments that mimic real conditions. For instance, oral drugs were tested against absorption through an environment similar to that of small intestine walls. ${ }^{54}$

Nanoemulsions have also been used as ultrasound imaging agents. ${ }^{117}$ Kaneda et al. prepared nanoemulsions containing perfluorocarbons for quantitative molecular imaging and targeted therapeutics. ${ }^{118}$ Gianella et al. ${ }^{115}$ engineered a multifunctional nanoemulsion based platform to enable an imaging-guided therapy (Fig. 9(b)). Researchers evaluated the utility of the platform in a colon cancer mouse model. In this study, oil-in-water nanoemulsions carried iron oxide nanocrystals for MRI, the fluorescent dye Cy7 for NIRF imaging, and the hydrophobic glucocorticoid prednisolone acetate valerate for therapeutic purposes.

\subsection{Nanoemulsions in food industry}

Nanoemulsions can be used in the food industry to design smart foods with ingredients that are otherwise difficult to 


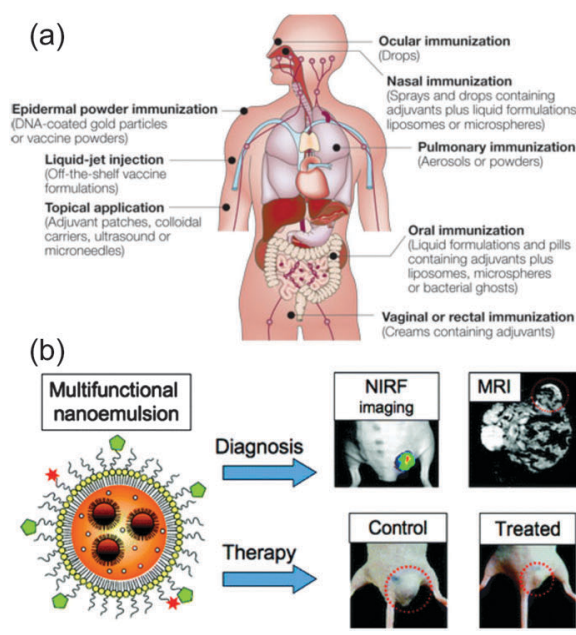

(c)
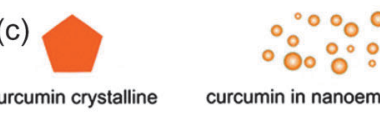

curcumin in nanoemulsions

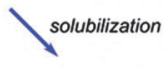

lipid digestion

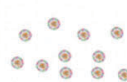

curcumin in mixed micelles

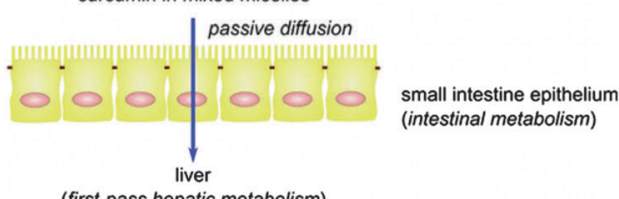

(first-pass hepatic metabolism)

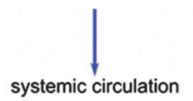

Fig. 9 Nanoemulsions for applications in drug delivery and the food industry. (a) Schematic of different modes of drug delivery in humans. Nanoemulsions have been tested for most of these modes as they significantly improve the bioavailability of hydrophobic drugs that are dissolved in the oil phase of O/W nanoemulsions. Figure reprinted with permission. ${ }^{114}$ Copyright (2005) by McMillan Publishers Ltd: Nature Reviews Immunology. (b) Magnetic nanoparticles added to nanoemulsions (that already consisted of hydrophobic drug in the oil phase) helps in the diagnosis (due to the presence of particles) and treatment of diseases. Figure reprinted with permission. ${ }^{115}$ Copyright (2011) by American Chemical Society. (c) Nanoemulsion with curcumin in the oil phase of nanoemulsion is easier to digest than when curcumin is taken directly due to easy lipid digestion step as compared to curcumin solubilization. ${ }^{70}$ Figure has been reprinted with permission. ${ }^{70}$ Copyright (2012) by American Chemical Society.

incorporate due to low-water solubility; an example is $\beta$-carotene, a pigment responsible for color in vegetables like carrots possessing important health benefits. Yuan et al. ${ }^{65,66}$ studied the size and stability of nanoemulsions with $\beta$-carotene against temperature, $\mathrm{pH}$ and surfactant type. Qian et al. ${ }^{6,7}$ prepared nanoemulsions with $\beta$-carotene and stabilized them with $\beta$-lactoglobulin, a biocompatible emulsifier. Researchers also reported the bioaccessibility of these nanoemulsions by simulating the oral, gastric and small intestine environments. $\beta$-Carotene nanoemulsions have been prepared with different methods (like high pressure homogenization, microfluidization and evaporative ripening) and different emulsifiers. . $^{6,64,67}$

Another additive that is quite often incorporated in nanoemulsions is curcumin, an anti-inflammatory agent. Wang et $a l^{69}$ explored the anti-inflammatory response of nanoemulsions through the mouse ear inflammatory model whereas Ahmed et al. ${ }^{68}$ explored the same using simulated digestion conditions. $\mathrm{Yu}$ and Huang ${ }^{70}$ have explored the possible application of nanoemulsions in improving the digestibility of food. The researchers showed that nanoemulsions prepared with circumin in the oil phase allow for easier digestion than when the circumin is taking in directly, owing to easy lipid digestion step in nanoemulsions (Fig. 9(c)).

Other studies on nanoemulsions in the food industry have explored the preparation and stability of flavored nanoemulsions using low energy methods. ${ }^{5,9-12,71-73}$ The use of nanoemulsions in the food industry has been covered extensively in two review articles and the interested reader is referred to these reports. ${ }^{4,22}$

\subsection{Nanoemulsions as building blocks}

Nanoemulsions can be used as building blocks for the preparation of more complex materials through exploitation of their small size and high surface area which enable easy decoration of a liquid-liquid surface with functional moieties such as designer macromolecules.

Emulsion polymerization is perhaps the best-known example in polymer synthesis where hydrophobic monomers contained in droplets are polymerized to create polymeric particles. Nanoemulsions (also known as miniemulsions) have been utilized extensively in polymer synthesis. ${ }^{78,120}$ More recently, Wu et al. ${ }^{79}$ synthesized compartmentalized silica nanospheres with structures like water-in-oil-in-silica-in-water with single or multiple water droplets in the innermost phase (see Fig. 10(a)). Oliveira et al. developed photoreactive surfactants for making nanoemulsions that consist of amphiphiles and also have the capability to act as reagents in photochemical reactions. ${ }^{121}$ Primo et al. prepared $\mathrm{O} / \mathrm{W}$ nanoemulsions in which the continuous phase consists of suspended magnetic nanoparticles. ${ }^{37}$ Chang et al. synthesized nanoemulsions with silicone oil as the dispersed phase and a mixture of sodium dodecyl sulphate and purified protein in water as the continuous phase. Upon emulsification, due to electrostatic interactions, virus like nanosized silicone oil droplets were coated with a layer of protein ${ }^{109}$ (see Fig. 10(b)). Researchers also showed that adjustment of the $\mathrm{pH}$ and ionic strength yielded different protein shell structures. Our group ${ }^{81}$ designed thermoresponsive nanoemulsions using photocurable polymer additives (see Fig. 10(c)). Upon crosslinking the composite hydrogels transformed into organogels with high storage modulus that are capable of carrying hydrophobic cargo and releasing the cargo. ${ }^{81}$ An et al.$^{80}$ synthesized composite hydrogels laden with high oxygen capacity nanoemulsions mimicking red blood cells (see Fig. 10(d)). They also demonstrated the controlled release of hydrophobic cargo from nanoemulsion-laden composite hydrogels, both through diffusion and disintegration of the hydrogel matrix (see Fig. 10(e)). 
(a)

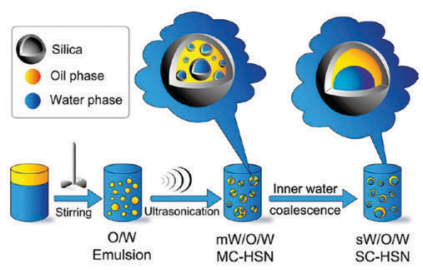

(c)

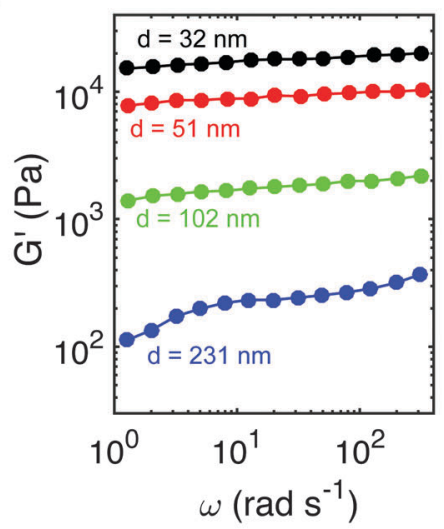

(b)

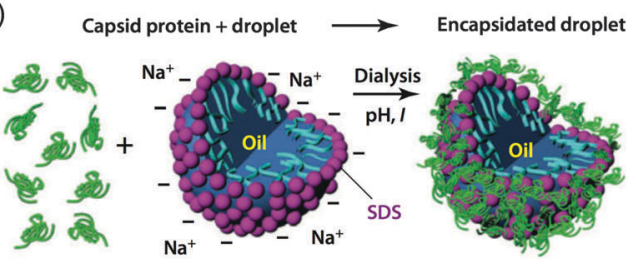

(d)

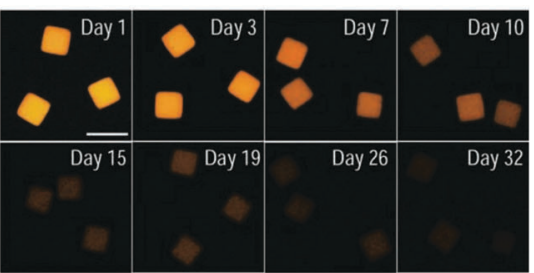

(e)

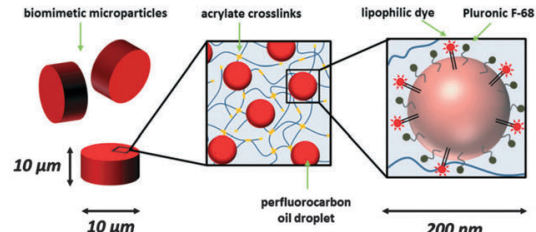

Fig. 10 Nanoemulsions as templates or building blocks for materials with unique properties. (a) Compartmentalized silica nanospheres have been synthesized using nanoemulsion droplets created by stirring and ultrasonification in a multi step synthesis. Figure adapted from Wu et al. ${ }^{79}$ Copyright (2013) by American Chemical Society. (b) Novel emulsions can be achieved through self-assembly of macromolecules on the surfaces of nanoemulsion droplets. Chang et al. ${ }^{109}$ encapsidated an oil droplet stabilized by anionic sodium dodecyl sulfate surfactant in water. Reprinted with permission. ${ }^{109}$ Copyright (2008) by American Chemical Society. (c) Nanoemulsions have been used in design of mesoporous, thermogelling and photocrosslinkable organohydrogels with very high modulus. The data is from Helgeson et al. ${ }^{81}$ (d) Nanoemulsions trapped in hydrogels have been used to demonstrate slow release of hydrophobic actives. Reprinted with permission. ${ }^{119}$ Copyrights (2012) by Wiley-VCH Verlag GmbH \& Co. KGaA, Weinheim. (e) Oxygen carrying nanoemulsions trapped in hydrogels have been designed to mimic artificial red blood cells. Adapted with permission. ${ }^{80}$ Copyrights (2013) by Royal Society of Chemistry.

\subsection{Nanoemulsions in crystallization/pharmaceuticals industry}

Eral et al. ${ }^{76,77}$ proposed an approach for producing size-controlled crystals of poorly water-soluble pharmaceutical compounds embedded in a polymer matrix through a soft matter inspired continuous process (Fig. 11(a)). This procedure eliminates the need for high energy grinding methods and avoids undesired
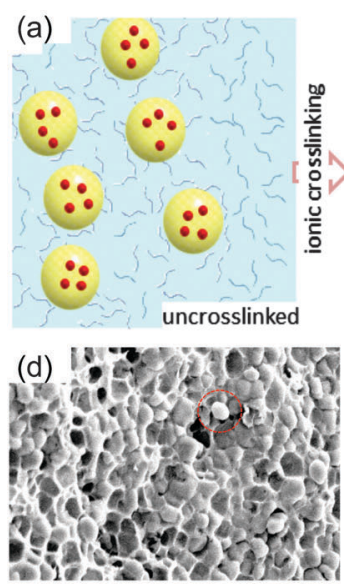
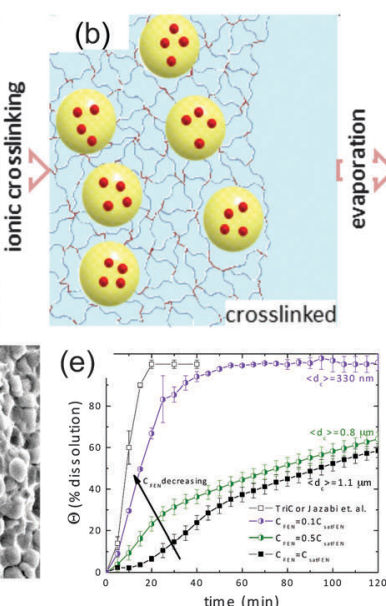
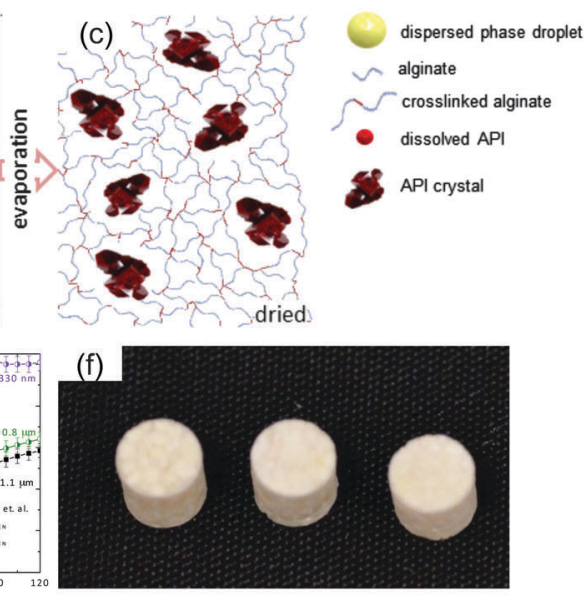

Fig. 11 Nanoemulsions in pharmaceutical crystallization. ${ }^{77}$ (a) Nanoemulsions trapped in a hydrogel matrix can be used to design an alternative processes that is energy and cost efficient and that avoids formation of undesired polymorphs. Nanoemulsion droplets carrying hydrophobic API are dispersed in an aqueous solvent containing uncrosslinked polymer alginate. (b) Upon crosslinking, the (nano- or macro-) emulsion droplets are entrapped in the elastic hydrogel matrix. (c) Following evaporation, dissolved API in emulsion droplets forms crystals that are embedded in the dried hydrogel matrix. (d) SEM image of dried composite hydrogel shows several crystals embedded in the hydrogel matrix, one highlighted with a red circle. (e) Dissolution profile of the model hydrophobic API fenofibrate with different crystal sizes dictated by the API concentration. The dissolution rate of $330 \mathrm{~nm}$ crystals is comparable to that of commercial formulations. (f) Tablets prepared from dried composite hydrogels. Figure adapted with permission from Eral et al. ${ }^{76}$ Copyright (2014) by American Chemical Society. 
polymorphic transitions, a major disadvantage of traditional schemes. The researchers dissolved the active pharmaceutical ingredient (API) in nanosized droplets of anisole in an aqueous medium containing alginate (a biopolymer) and F68 (a biocompatible polymeric surfactant); and cross-linked the continuous phase leaving a droplets trapped in a hydrogel. The resulting soft material is a composite hydrogel (Fig. 11(b)). Through evaporation of the composite hydrogel, authors showed that crystals of controlled size (330 $\mathrm{nm}-420 \mu \mathrm{m}$ ) and loading (up to $85 \% \mathrm{w} / \mathrm{w}$ ) can be produced (Fig. 11(c) and (d)). Eral et al. ${ }^{76,77}$ also demonstrated that dissolution rates comparable to commercially available formulations of fenofibrate can be achieved (Fig. 11(e)). Furthermore, dried composite hydrogels can be produced with a continuous process and the final formulation containing the API and biocompatible polymer alginate can be compressed into tablets (Fig. 11(f)).

\section{Outlook}

Nanoemulsions have gained popularity over the past decade because of their exceptional properties such as high surface area, transparent appearance, robust stability and tunable rheology. The most widely used preparation methods for nanoemulsions include high energy methods such as high pressure homogenization and ultrasonication, and low energy methods such as phase inversion temperature and emulsion inversion point. Emergent synthesis techniques are bubble bursting at liquid/air interface, evaporative ripening and microfluidization. There is little understanding of the possible industrial relevance of many of these approaches as the physics of nanoemulsion formation is still semi-empirical and rational scale-up procedure have not been widely explored.

Nanoemulsions have been shown to exhibit robust stability with research focusing on the study of different parameters to lower the rate of Ostwald ripening. As discussed in this article, strategies such as trapped species methods have been developed to make stable nanoemulsions. However, current research has not explored the idea of designing polymers that can be used as emulsifier for the preparation of stable nanoemulsions. Polymers can be tuned from being hydrophobic to hydrophillic which can result in rich properties, including tunable rheology and stability. Due to their small size, molecules sitting at the interface of nanoemulsions experience higher curvature which will greatly influence self-assembly at the interface. Nanoemulsions can also serve as a model system to enhance understanding of colloidal assembly and rheology of complex emulsion systems. They can be readily density matched, selectively dyed for visualization, and made magnetic field responsive. Their liquid interfaces are dynamic, which expands the richness of their soft matter physical properties.

Leveraging the high surface area of nanoemulsions, researchers have used them extensively in drug delivery, and in the food, cosmetics and the pharmaceutical industries. While nanoemulsion applications within these industries look promising and there is a need for continued research in these areas, many other potential uses, such as enhanced oil recovery ${ }^{122}$ or tissue engineering, are relatively unexplored.

\section{Acknowledgements}

The authors would like to thank Eni SPA for providing funds for this project.

\section{References}

1 T. G. Mason, J. Wilking, K. Meleson, C. Chang and S. Graves, J. Phys.: Condens. Matter, 2006, 18, R635.

2 T. Delmas, H. Piraux, A.-C. Couffin, I. Texier, F. Vinet, P. Poulin, M. E. Cates and J. Bibette, Langmuir, 2011, 27, 1683-1692.

3 D. J. McClements, Soft Matter, 2011, 7, 2297-2316.

4 D. J. McClements and J. Rao, Crit. Rev. Food Sci. Nutr., 2011, 51, 285-330.

5 F. Ostertag, J. Weiss and D. J. McClements, J. Colloid Interface Sci., 2012, 388, 95-102.

6 C. Qian, E. A. Decker, H. Xiao and D. J. McClements, Food Chem., 2012, 135, 1440-1447.

7 C. Qian, E. A. Decker, H. Xiao and D. J. McClements, Food Chem., 2012, 132, 1221-1229.

8 C. Qian and D. J. McClements, Food Hydrocolloids, 2011, 25, 1000-1008.

9 J. Rao and D. J. McClements, J. Agric. Food Chem., 2011, 59, 5026-5035.

10 J. Rao and D. J. McClements, Food Hydrocolloids, 2011, 25, 1413-1423.

11 J. Rao and D. J. McClements, J. Agric. Food Chem., 2010, 58, 7059-7066.

12 E. Troncoso, J. M. Aguilera and D. J. McClements, Food Hydrocolloids, 2012, 27, 355-363.

13 T. Tadros, P. Izquierdo, J. Esquena and C. Solans, Adv. Colloid Interface Sci., 2004, 108, 303-318.

14 C. Solans, P. Izquierdo, J. Nolla, N. Azemar and M. GarciaCelma, Curr. Opin. Colloid Interface Sci., 2005, 10, 102-110.

15 M. M. Fryd and T. G. Mason, Annu. Rev. Phys. Chem., 2012, 63, 493-518.

16 P. Izquierdo, J. Esquena, T. F. Tadros, C. Dederen, M. Garcia, N. Azemar and C. Solans, Langmuir, 2002, 18, 26-30.

17 A. Forgiarini, J. Esquena, C. Gonzalez and C. Solans, Trends Colloid Interface Sci. XIV, 2000, 36-39.

18 A. Forgiarini, J. Esquena, C. González and C. Solans, Trends Colloid Interface Sci. XV, 2001, 184-189.

19 A. Forgiarini, J. Esquena, C. Gonzalez and C. Solans, Langmuir, 2001, 17, 2076-2083.

20 J. Feng, M. Roch, D. Vigolo, L. N. Arnaudov, S. D. Stoyanov, T. D. Gurkov, G. G. Tsutsumanova and H. A. Stone, Nat. Phys., 2014, 10, 606-612.

21 M. M. Fryd and T. G. Mason, J. Phys. Chem. Lett., 2010, 1, 3349-3353.

22 D. J. McClements, Soft Matter, 2012, 8, 1719-1729. 
23 N. Anton and T. F. Vandamme, Pharm. Res., 2011, 28, 978-985.

24 P. Izquierdo, J. Feng, J. Esquena, T. F. Tadros, J. C. Dederen, M. J. Garcia, N. Azemar and C. Solans, J. Colloid Interface Sci., 2005, 285, 388-394.

25 I. Solè, A. Maestro, C. Pey, C. González, C. Solans and J. M. Gutiérrez, Colloids Surf., A, 2006, 288, 138-143.

26 P. Izquierdo, J. Esquena, T. F. Tadros, J. C. Dederen, J. Feng, M. J. Garcia-Celma, N. Azemar and C. Solans, Langmuir, 2004, 20, 6594-6598.

27 N. Uson, M. J. Garcia and C. Solans, Colloids Surf., A, 2004, 250, 415-421.

28 M. Porras, C. Solans, C. Gonzalez and J. Gutierrez, Colloids Surf., A, 2008, 324, 181-188.

29 M. Porras, C. Solans, C. Gonzalez, A. Martnez, A. Guinart and J. M. Gutiérrez, Colloids Surf., A, 2004, 249, 115-118.

30 S. Baboota, F. Shakeel, A. Ahuja, J. Ali and S. Shafiq, Acta Pharm., 2007, 57, 315-332.

31 N. Dixit, K. Kohli and S. Baboota, PDA J. Pharm. Sci. Technol., 2008, 62, 46-55.

32 M. Kong, X. G. Chen, D. K. Kweon and H. J. Park, Carbohydr. Polym., 2011, 86, 837-843.

33 F. Shakeel, S. Baboota, A. Ahuja, J. Ali, M. Aqil and S. Shafiq, AAPS PharmSciTech, 2007, 8, 191-199.

34 F. Shakeel and W. Ramadan, Colloids Surf., B, 2010, 75, 356-362.

35 P. Alves, A. Pohlmann and S. Guterres, Pharmazie, 2005, 60, 900-904.

36 D. Mou, H. Chen, D. Du, C. Mao, J. Wan, H. Xu and X. Yang, Int. J. Pharm., 2008, 353, 270-276.

37 F. L. Primo, L. Michieleto, M. A. Rodrigues, P. P. Macaroff, P. C. Morais, Z. G. Lacava, M. V. L. Bentley and A. C. Tedesco, J. Magn. Magn. Mater., 2007, 311, 354-357.

38 H. Wu, C. Ramachandran, N. D. Weiner and B. J. Roessler, Int. J. Pharm., 2001, 220, 63-75.

39 H. Zhou, Y. Yue, G. Liu, Y. Li, J. Zhang, Q. Gong, Z. Yan and M. Duan, Nanoscale Res. Lett., 2010, 5, 224-230.

40 B. S. Kim, M. Won, K. M. Lee and C. S. Kim, Drug Delivery, 2008, 15, 465-469.

41 E. Yilmaz and H.-H. Borchert, Int. J. Pharm., 2006, 307, 232-238.

42 E. Yilmaz and H.-H. Borchert, Eur. J. Pharm. Biopharm., 2005, 60, 91-98.

43 V. Klang, N. Matsko, A.-M. Zimmermann, E. Vojnikovic and C. Valenta, Int. J. Pharm., 2010, 393, 153-161.

44 F. Shakeel, S. Baboota, A. Ahuja, J. Ali, M. Faisal and S. Shafiq, Thai J. Pharm. Sci., 2008, 32, 4-9.

45 V. B. Junyaprasert, V. Teeranachaideekul, E. B. Souto, P. Boonme and R. H. Müller, Int. J. Pharm., 2009, 377, 207-214.

46 J.-B. Tagne, S. Kakumanu and R. J. Nicolosi, Mol. Pharmaceutics, 2008, 5, 1055-1063.

47 J.-B. Tagne, S. Kakumanu, D. Ortiz, T. Shea and R. J. Nicolosi, Mol. Pharmaceutics, 2008, 5, 280-286.

48 H. Ammar, H. Salama, M. Ghorab and A. Mahmoud, Drug Dev. Ind. Pharm., 2010, 36, 1330-1339.
49 F. Lallemand, P. Daull, S. Benita, R. Buggage and J.-S. Garrigue, J. Drug Delivery, 2012, 2012, 1-16.

50 H. O. Ammar, H. Salama, M. Ghorab and A. Mahmoud, AAPS PharmSciTech, 2009, 10, 808-819.

51 M. Ahmed, W. Ramadan, D. Rambhu and F. Shakeel, Pharmazie, 2008, 63, 806-811.

52 M. Kumar, A. Misra, A. Babbar, A. Mishra, P. Mishra and K. Pathak, Int. J. Pharm., 2008, 358, 285-291.

53 M. Kumar, A. Misra, A. Mishra, P. Mishra and K. Pathak, J. Drug Targeting, 2008, 16, 806-814.

54 C. Brüsewitz, A. Schendler, A. Funke, T. Wagner and R. Lipp, Int. J. Pharm., 2007, 329, 173-181.

55 R. G. Kelmann, G. Kuminek, H. F. Teixeira and L. S. Koester, Int. J. Pharm., 2007, 342, 231-239.

56 S. Shafiq-un Nabi, F. Shakeel, S. Talegaonkar, J. Ali, S. Baboota, A. Ahuja, R. K. Khar and M. Ali, AAPS PharmSciTech, 2007, 8, E12-E17.

57 K. K. Singh and S. K. Vingkar, Int. J. Pharm., 2008, 347, 136-143.

58 S. Shafiq and F. Shakeel, AAPS PharmSciTech, 2008, 9, 1097-1101.

59 T. K. Vyas, A. Shahiwala and M. M. Amiji, Int. J. Pharm., 2008, 347, 93-101.

60 S. Calderilla-Fajardo, J. Cazares-Delgadillo, R. VillalobosGarcia, D. Quintanar-Guerrero, A. Ganem-Quintanar and R. Robles, Drug Dev. Ind. Pharm., 2006, 32, 107-113.

61 N. Anton, P. Gayet, J.-P. Benoit and P. Saulnier, Int. J. Pharm., 2007, 344, 44-52.

62 S. C. de Araújo, A. C. A. de Mattos, H. F. Teixeira, P. M. Z. Coelho, D. L. Nelson and M. C. de Oliveira, Int. J. Pharm., 2007, 337, 307-315.

63 L. Mao, D. Xu, J. Yang, F. Yuan, Y. Gao and J. Zhao, Food Technol. Biotechnol., 2009, 47, 336-342.

64 L. Mao, J. Yang, D. Xu, F. Yuan and Y. Gao, J. Dispersion Sci. Technol., 2010, 31, 986-993.

65 Y. Yuan, Y. Gao, L. Mao and J. Zhao, Food Chem., 2008, 107, 1300-1306.

66 Y. Yuan, Y. Gao, J. Zhao and L. Mao, Food Res. Int., 2008, 41, 61-68.

67 H. D. Silva, M. A. Cerqueira, B. W. Souza, C. Ribeiro, M. C. Avides, M. A. Quintas, J. S. Coimbra, M. G. Carneiro-da Cunha and A. A. Vicente, J. Food Eng., 2011, 102, 130-135.

68 K. Ahmed, Y. Li, D. J. McClements and H. Xiao, Food Chem., 2012, 132, 799-807.

69 X. Wang, Y. Jiang, Y.-W. Wang, M.-T. Huang, C.-T. Ho and Q. Huang, Food Chem., 2008, 108, 419-424.

$70 \mathrm{H}$. Yu and Q. Huang, J. Agric. Food Chem., 2012, 60, 5373-5379.

71 S. M. Jafari, Y. He and B. Bhandari, Eur. Food Res. Technol., 2007, 225, 733-741.

72 S. Kentish, T. Wooster, M. Ashokkumar, S. Balachandran, R. Mawson and L. Simons, Innovative Food Sci. Emerging Technol., 2008, 9, 170-175.

73 S. J. Lee and D. J. McClements, Food Hydrocolloids, 2010, 24, 560-569. 
74 O. Sonneville-Aubrun, J.-T. Simonnet and F. L'alloret, Adv. Colloid Interface Sci., 2004, 108, 145-149.

75 U. Sakulku, O. Nuchuchua, N. Uawongyart, S. Puttipipatkhachorn, A. Soottitantawat and U. Ruktanonchai, Int. J. Pharm., 2009, 372, 105-111.

76 H. B. Eral, V. López-Mejas, M. O’Mahony, B. L. Trout, A. S. Myerson and P. S. Doyle, Cryst. Growth Des., 2014, 14, 2073-2082.

77 H. B. Eral, M. O’Mahony, R. Shaw, B. L. Trout, A. S. Myerson and P. S. Doyle, Chem. Mater., 2014, 26, 6213-6220.

78 J. M. Asua, Prog. Polym. Sci., 2002, 27, 1283-1346.

79 S.-H. Wu, Y. Hung and C.-Y. Mou, Chem. Mater., 2013, 25, 352-364.

80 H. Z. An, E. R. Safai, H. B. Eral and P. S. Doyle, Lab Chip, 2013, 13, 4765-4774.

81 M. E. Helgeson, S. E. Moran, H. Z. An and P. S. Doyle, Nat. Mater., 2012, 11, 344-352.

82 J. Davies, Chem. Eng. Sci., 1985, 40, 839-842.

83 J. Davies, Chem. Eng. Sci., 1987, 42, 1671-1676.

84 A. Gupta, H. B. Eral, T. A. Hatton and P. S. Doyle, Soft Matter, 2016, 12, 1452-1458.

85 J. Hinze, AIChE J., 1955, 1, 289-295.

86 K. Meleson, S. Graves and T. G. Mason, Soft Mater., 2004, 2, 109-123.

87 T. J. Wooster, M. Golding and P. Sanguansri, Langmuir, 2008, 24, 12758-12765.

88 J. Floury, J. Bellettre, J. Legrand and A. Desrumaux, Chem. Eng. Sci., 2004, 59, 843-853.

89 T. Mason, S. Graves, J. Wilking and M. Lin, Condens. Matter Phys., 2006, 9, 193-199.

90 T. Gothsch, J. H. Finke, S. Beinert, C. Lesche, J. Schur, S. Buettgenbach, C. Müller-Goymann and A. Kwade, Chem. Eng. Technol., 2011, 34, 335-343.

91 P. Becher, Encyclopedia of Emulsion Technology: Vol. 1 Basic Theory, Marcel Dekker, 1983.

92 F. Dons, M. Annunziata, M. Vincensi and G. Ferrari, J. Biotechnol., 2012, 159, 342-350.

93 F. Dons, M. Sessa and G. Ferrari, Ind. Eng. Chem. Res., 2011, 51, 7606-7618.

94 C. Pey, A. Maestro, I. Solé, C. González, C. Solans and J. M. Gutiérrez, Colloids Surf., A, 2006, 288, 144-150.

95 I. Solè, A. Maestro, C. González, C. Solans and J. M. Gutiérrez, Langmuir, 2006, 22, 8326-8332.

96 D. Morales, J. M. Gutiérrez, M. Garcia-Celma and Y. Solans, Langmuir, 2003, 19, 7196-7200.

97 S. Liedtke, S. Wissing, R. Müller and K. Mäder, Int. J. Pharm., 2000, 196, 183-185.

98 T. Leong, T. Wooster, S. Kentish and M. Ashokkumar, Ultrason. Sonochem., 2009, 16, 721-727.

99 S. M. Jafari, E. Assadpoor, Y. He and B. Bhandari, Food Hydrocolloids, 2008, 22, 1191-1202.
100 S. M. Jafari, Y. He and B. Bhandari, J. Food Eng., 2007, 82, 478-488.

101 J. M. Yuk, J. Park, P. Ercius, K. Kim, D. J. Hellebusch, M. F. Crommie, J. Y. Lee, A. Zettl and A. P. Alivisatos, Science, 2012, 336, 61-64.

102 D. Conchouso, D. Castro, S. A. Khan and I. G. Foulds, Lab Chip, 2014, 14, 3011-3020.

103 K. Rahn-Chique, A. M. Puertas, M. S. Romero-Cano, C. Rojas and G. Urbina-Villalba, Adv. Colloid Interface Sci., 2012, 178, 1-20.

104 I. M. Lifshitz and V. V. Slyozov, J. Phys. Chem. Solids, 1961, 19, 35-50.

105 J. Mewis and N. J. Wagner, Colloidal suspension rheology, Cambridge University Press, 2012.

106 T. Tadros, Adv. Colloid Interface Sci., 2004, 108, 227-258.

107 W. B. Russel, D. A. Saville and W. R. Schowalter, Colloidal dispersions, Cambridge University Press, 1992.

108 M. Porras, A. Martnez, C. Solans, C. González and J. M. Gutiérrez, Colloids Surf., A, 2005, 270, 189-194.

109 C. B. Chang, C. M. Knobler, W. M. Gelbart and T. G. Mason, ACS Nano, 2008, 2, 281-286.

110 J. N. Wilking and T. G. Mason, Phys. Rev. E: Stat., Nonlinear, Soft Matter Phys., 2007, 75, 041407.

111 Y. Gao, J. Kim and M. E. Helgeson, Soft Matter, 2015, 11, 6360-6370.

112 L. C. Hsiao and P. S. Doyle, Soft Matter, 2015, 11, 8426-8431.

113 M. E. Helgeson, Y. Gao, S. E. Moran, J. Lee, M. Godfrin, A. Tripathi, A. Bose and P. S. Doyle, Soft Matter, 2014, 10, 3122-3133.

114 S. Mitragotri, Nat. Rev. Immunol., 2005, 5, 905-916.

115 A. Gianella, P. A. Jarzyna, V. Mani, S. Ramachandran, C. Calcagno, J. Tang, B. Kann, W. J. Dijk, V. L. Thijssen and A. W. Griffioen, et al., ACS Nano, 2011, 5, 4422-4433.

116 S. Shafiq, F. Shakeel, S. Talegaonkar, F. J. Ahmad, R. K. Khar and M. Ali, Eur. J. Pharm. Biopharm., 2007, 66, 227-243.

117 N. Rapoport, K.-H. Nam, R. Gupta, Z. Gao, P. Mohan, A. Payne, N. Todd, X. Liu, T. Kim and J. Shea, et al., J. Controlled Release, 2011, 153, 4-15.

118 M. M. Kaneda, S. Caruthers, G. M. Lanza and S. A. Wickline, Ann. Biomed. Eng., 2009, 37, 1922-1933.

119 H. Z. An, M. E. Helgeson and P. S. Doyle, Adv. Mater., 2012, 24, 3838-3844.

120 K. Landfester, Annu. Rev. Mater. Res., 2006, 36, 231-279.

121 R. J. de Oliveira, P. Brown, G. B. Correia, S. E. Rogers, R. Heenan, I. Grillo, A. Galembeck and J. Eastoe, Langmuir, 2011, 27, 9277-9284.

122 L. Del Gaudio, C. R. Pratesi, A. Belloni and I. Moroni, Process for the recovery of heavy oil from an underground reservoir, US Pat. App. 13/503,033, 2010. 